\title{
Molecular resolvent-operator method: Electronic and nuclear dynamics in strong-field ionization
}

\author{
F. Catoire,,${ }^{1, *}$ R. E. F. Silva, ${ }^{2}$ P. Rivière, ${ }^{2}$ H. Bachau, ${ }^{1}$ and F. Martín ${ }^{2,3}$ \\ ${ }^{1}$ Centre des Lasers Intenses et Applications, UMR 5107 CNRS-CEA-Université de Bordeaux, \\ 351 Cours de la Libération, Talence F-33405, France \\ ${ }^{2}$ Departamento de Química, Módulo 13, Universidad Autónoma de Madrid, 28049 Madrid, Spain \\ ${ }^{3}$ Instituto Madrileño de Estudios Avanzados en Nanociencia, Cantoblanco, 28049 Madrid, Spain
}

(Received 22 July 2013; published 13 February 2014)

\begin{abstract}
We present an extension of the resolvent-operator method (ROM), originally designed for atomic systems, to extract differential photoelectron spectra (in photoelectron- and nuclear-kinetic energy) for diatomic molecules interacting with strong, ultrashort laser fields in the single active electron approximation. The method is applied to the study of $\mathrm{H}_{2}{ }^{+}$photodissociation and photoionization by femtosecond laser pulses in the XUV-IR frequency range. In particular, the method is tested (i) in the perturbative regime, for few-photon absorption and bound-bound electronic transitions, and (ii) in the strong-field regime, in which multiphoton absorption and tunneling are present. In the latter case, we show how the differential ROM allows one to track the transition between both regimes. We also analyze isotopic effects by comparing the dynamics of $\mathrm{H}_{2}{ }^{+}$and $\mathrm{D}_{2}{ }^{+}$ionization for different pulses.
\end{abstract}

DOI: 10.1103/PhysRevA.89.023415

PACS number(s): 33.20.Xx, 33.80.Rv, 33.60.+q

\section{INTRODUCTION}

When a molecular system interacts with an intense $\left(I>10^{13} \mathrm{~W} / \mathrm{cm}^{2}\right)$ infrared (IR) laser field, nonperturbative phenomena such as high-harmonic generation and abovethreshold ionization (ATI) can occur. Several new concepts have arisen from the understanding of molecular dissociation and ionization in strong fields, such as bond softening [1], bond hardening [2], or charge-resonance-enhanced ionization (CREI) [3]. In particular, the hydrogen molecule and its molecular ion, due to their simplicity, have been the subject of many theoretical and experimental works over the years [4-6].

The analysis of molecular dynamics is usually done through three observables: high-harmonic generation (HHG) $[7,8]$, the kinetic energy release (KER) of the molecular target [9], and the photoelectron-kinetic energy spectrum (EKE) [10]. HHG occurs when the laser is strong enough to ionize the molecular (or atomic) target and drives the escaping electron so that the latter may eventually recombine with the ion, leading to the emission of high-energy photons, usually in the UV to XUV frequency range [11]. As one of the properties of these harmonics is coherence, the harmonic spectrum contains information about the molecular structure and the induced dynamics $[12,13]$. The measurement of the kinetic energy of the electrons that escape forever (i.e., do not recombine) and the associated molecular cation (EKE and KER) allows one to study time-dependent processes such as the nuclear dynamics in, e.g., $\mathrm{H}_{2}$ [14,15] or $\mathrm{H}_{2}{ }^{+}$[16-19]. The KER spectra for $\mathrm{H}_{2}{ }^{+}$ionization show peculiar structures, for which different mechanisms, such as CREI or above-threshold Coulomb explosion, have been proposed [20-23]. In a similar context, electron rescattering is suspected to play a major role for high-energy peaks observed in the KER spectrum [24].

From the theoretical point of view, the models that have been used to analyze these phenomena have often been applied to a "frozen" molecule (i.e., with fixed internuclear distance) [25]. The Born-Oppenheimer (BO) approximation,

*catoire@celia.u-bordeaux1.fr which assumes that the electronic and nuclear motions can be separated, has been widely used (see, e.g., [26-29]). However, there are cases in which this approximation is not valid and electron and nuclear dynamics cannot be disentangled [30]. This occurs, for instance, when slow electrons are released with a velocity comparable to that of the nuclei, or in molecular autoionization, where the nuclei have enough time to move before electrons are ejected. In such cases, both electron and nuclear dynamics must be considered on an equal footing, and instead of measuring photoelectron and ion spectra separately, it is much more appropriate to measure electrons and ions in coincidence [31], which is now experimentally feasible [32]. The measured ionization yield plotted as a function of both the electron- and ion-kinetic energies [hereafter called for short correlated kinetic energy (CKE) spectrum] thus exhibits the electronic and nuclear dynamics, as well as the connection between them $[14,15,19,33]$. While most theoretical works on molecules interacting with strong IR fields have reported either KER or EKE spectra, recently, few works (experimental and theoretical) have shown CKE spectra resulting from $\mathrm{H}_{2}{ }^{+}$ and $\mathrm{H}_{2}$ photoionization $[16,19,34]$. These CKE spectra show clearly how the energy is shared between the electron and the protons. Thus, combining coincidence experiments and theoretical CKE spectra, it will be soon possible to get a deeper insight into the mechanisms involved in molecular ionization.

There are two main approaches to solve numerically the time-dependent Schrödinger equation (TDSE) for atomic or molecular photoionization. The first includes spectral methods, in which the wave function is expressed as a linear combination of eigenstates of the field-free Hamiltonian, thus leading to a set of first-order coupled differential equations [35]. This approach requires to obtain in advance the dipole matrix elements for every possible initial and final state, and, in most applications, it avoids the evaluation of nonadiabatic couplings. Due to limitations in the number of states that one can include in the expansion, most calculations are performed in the XUV regime. Alternatively, the TDSE can be solved numerically on a discretized spatial grid in the so-called grid method [36]. In the molecular case, the solution of TDSE without resorting to the $\mathrm{BO}$ approximation does not bring 
any additional complexity. However, and unlike in spectral methods, the extraction of observables is not a trivial task. A method for obtaining accurate kinetic energy spectra in gridbased calculations is the resolvent-operator method (ROM), initially applied in atomic photoionization by Schafer and Kulander [37] and recently improved to obtain absolute density probabilities [38]. In previous work [38], it has been shown, for the case of the $\mathrm{H}$ atom studied in its full dimensionality, that results obtained by using the atomic version of the ROM are in excellent agreement with those obtained from elaborate spectral methods in which the wave function is expanded in a basis of atomic eigenstates and the ionization probabilities are obtained by projecting on scattering states.

Due to the large number of particles involved in molecular calculations, the single active electron (SAE) approximation is usually invoked when only one electron is relevant for the dynamics $[39,40]$. Here we focus on the monoelectronic $\mathrm{H}_{2}{ }^{+}$ molecular ion. In this work we describe the extension of the ROM to diatomic molecules in the framework of the SAE. A few illustrative results of this method, obtained for the case of intense IR pulses, have been recently published [16]. In this work we present the detailed mathematical foundations of the ROM and perform a systematic study of the CKE spectra that result from $\mathrm{H}_{2}{ }^{+}$ionization by intense ultrashort laser pulses in a wide range of photon energies (from the IR to the XUV). More specifically, the applications focus on (i) one-photon ionization, (ii) resonant enhanced multiphoton ionization (REMPI), and (iii) ionization of $\mathrm{H}_{2}{ }^{+}$and $\mathrm{D}_{2}{ }^{+}$in the so-called tunneling regime. We also discuss the accuracy that one can expect to reach by using different versions of the $\mathrm{ROM}$, as well as the validity of the BO approximation.

Atomic units (a.u.) are used throughout the paper unless stated otherwise.

\section{THEORETICAL FRAMEWORK}

The energy analysis of the final wave function that results from the interaction of a laser pulse with a molecular system can be performed in different ways, for example, through a projection onto field-free eigenstates or calculating the time evolution of the autocorrelation function of the propagated wave function and obtaining the energy spectrum from its Fourier transform. The first method requires the calculation of all eigenstates, including those in the continuum, which is extremely demanding for high-dimensional systems. In that case, the use of the spectral method is more reasonable, since it directly provides the time-dependent population of the eigenstates. The autocorrelation method [41], on the other hand, only provides access to the total energy of the system. For atomic systems this might be enough, but in the case of molecular systems one may need to know the energy spectra of the individual fragments.

A recently proposed alternative to those two methods is the ROM for molecules [16]. From the ROM one can extract the differential energy spectra for electrons and nuclei, or, as we show here, the absolute total probability density as a function of the total energy of the system.

In this work we restrict our study to the case of diatomic molecules described within the SAE, i.e., to two fragments: the outgoing electron and the center of mass of the nuclei.
We also assume that the rotation time of the molecule is too long to be accounted for, so that the rotational component of the Hamiltonian can be dropped out. The Hamiltonian of the system under consideration is then written

$$
\hat{H}_{0}=\hat{T}_{N}+\hat{H}_{e l},
$$

where $\hat{T}_{N}=-\frac{1}{2} \sum_{i} \frac{1}{\mu_{i}} \hat{\nabla}_{\mathbf{R}_{\mathbf{i}}}^{2}$ is the kinetic operator associated with the nuclear motion, which includes the nuclear coordinates $\left(\mathbf{R}_{\mathbf{i}}\right)$ in the center-of-mass frame. The electronic Hamiltonian is $\hat{H}_{e l}=\hat{T}_{e l}+\hat{V}_{e N}+\hat{V}_{i}(\mathbf{R})$, where $\hat{T}_{e l}=-\frac{1}{2} \hat{\nabla}_{\mathbf{r}}^{2}$ is the electron-kinetic energy, and $\hat{V}_{i}(\mathbf{R})$ describes the nucleusnucleus interaction. In the case of the $\mathrm{H}_{2}{ }^{+}$molecule, $\hat{V}_{i}(\mathbf{R})=$ $1 / R$. Finally, $\hat{V}_{e N}$ is the electron-nuclear interaction.

In this work we have solved numerically the TDSE in a two-dimensional (2D) grid $(z, R)$, where $z$ is the electronic coordinate, parallel to the linear polarization axis of the laser field, and $R$ is the internuclear distance, for different laser pulses. We have used a soft-core potential $\hat{V}_{e N}(z, R)$ to avoid the Coulomb singularity [42]. The total Hamiltonian is $\hat{H}=$ $\hat{H}_{0}+\hat{V}(z, t)$, where $\hat{V}(z, t)=\hat{z} \hat{E}(t)$ is the laser potential in length gauge. We refer to this model of the $\mathrm{H}_{2}{ }^{+}$molecule as $(1+1) \mathrm{D}$ model.

We have used a box with $|z|<1500$ a.u. and $R<30$ a.u., with uniform grid spacings of $\Delta z=0.1$ a.u. and $\Delta R=$ 0.05 a.u. The propagation was performed by using the CrankNicolson split-operator method with $\Delta t=0.02$ a.u. We have checked that by increasing the electronic box size by $13 \%$, the nuclear box size by $17 \%$, and the density of the spatial and temporal grids by $50 \%$, the results do not change (see Appendix B). The laser pulses have a photon energy $\Omega$, a total pulse duration $T$, and a peak intensity $I$. A $\sin ^{2}$ envelope was used in all cases. The propagation is performed until the end of the pulse or later, when the required observables are extracted with the resolvent-operator method (ROM).

In Sec. III A we describe how to use the ROM to extract the total energy of the Hamiltonian given by Eq. (1). In Sec. III B we show how to extract the differential probability density by means of the ROM. In Sec. III C we explain the physical interpretation of the method, in Sec. IIID we focus on the normalization issue, and finally, in Sec. III E, we describe the link between the total ROM and the differential ROM.

It must be noticed that the TDSE propagation, as well as the total ROM calculations, are performed without making use of the BO approximation. In the differential ROM, which provides differential electron- and nuclear-kinetic energy spectra, the BO approximation is used for the projection, but not for the time propagation. This procedure in the projection is unavoidable since the TDSE cannot be integrated up to infinite time. The accuracy of the BO approximation used in the differential ROM is also discussed.

\section{DESCRIPTION OF THE RESOLVENT-OPERATOR METHOD}

\section{A. The total energy distribution}

Let $\Psi_{f}(\mathbf{r}, \mathbf{R})$ be the wave function that is obtained at the end of the pulse by solving the TDSE. In the same way we note $\Psi_{k}(\mathbf{r}, \mathbf{R})=\left\langle\mathbf{r}, \mathbf{R} \mid \Psi_{k}\right\rangle$ the eigenstates of the Hamiltonian 
$\hat{H}_{0}$ so that

$$
\hat{H}_{0}\left|\Psi_{k}\right\rangle=E_{k}\left|\Psi_{k}\right\rangle \text {. }
$$

We define the resolvent operator of degree $n$ as

$$
\hat{R}_{ \pm}=\frac{\delta^{n}}{\left(E-\hat{H}_{0}\right)^{n} \pm i \delta^{n}} .
$$

Either $\hat{R}_{+}$or $\hat{R}_{-}$can be used indifferently without changing the physical insight, so we will simply note the resolvent operator as $\hat{R}$. In Eq. (3), $E$ refers to the total energy and $\delta$ to a parameter associated with the energy resolution [38]. The resolvent operator extracts from the wave function a particular region of the energy spectrum centered around $E$ with an energy width $\delta$. The definition of this operator is then similar to the atomic case and all properties associated with this resolvent can be applied to the molecular case [38]. By defining

$$
P(E, \delta)=\left\langle\Psi_{f}\left|\hat{R}^{\dagger} \hat{R}\right| \Psi_{f}\right\rangle,
$$

it can be shown that, for a nondegenerate bound state $\Psi_{k}$ and a small-enough $\delta, P\left(E_{k}, \delta\right)=\left|c_{k}\right|^{2}$ where $c_{k}=\left\langle\Psi_{k} \mid \Psi_{f}\right\rangle$. Hence, $P\left(E_{k}, \delta\right)$ is the probability of finding our system with energy $E_{k}$ at the end of the pulse. If $E$ belongs to the continuum spectrum of $\hat{H}_{0}$, then the probability density is given by

$$
\rho(E)=\frac{n P(E, \delta)}{\delta \pi \csc \left(\frac{\pi}{2 n}\right)},
$$

which is independent of $\delta$ if the latter is chosen properly, i.e., close to the density of states in the continuum defined by the discretization [38]. In practice, the resolvent $(\hat{R})$ is defined by the Green's function $\hat{G}$ such that

$$
\begin{aligned}
\frac{\delta^{n}}{\left(E-\hat{H}_{0}\right)^{n} \pm i \delta^{n}} & =\prod_{j=1}^{n} \delta \hat{G}\left(E-q_{j} \delta\right), \\
\hat{G}\left(E-q_{j} \delta\right) & =\frac{1}{E-\hat{H}_{0}-q_{j} \delta},
\end{aligned}
$$

where $q_{j}=i^{1 / n} z_{j}$ and $z_{j}$ is the $j$ th choice of the $n$th root of unity. The use of the Green's operator transforms the resolvent into an operator which depends linearly on the Hamiltonian $[37,38]$. The application of the Green's function on the final wave function is then reduced to an inversion problem (see more details in Appendix A). From this method only the total energy can be extracted, but it is self-consistent in the sense that no approximations are performed and no basis is used (which would be necessarily truncated).

\section{B. Differential ROM: Correlated electron- and nuclear-energy spectra}

If one wants to disentangle the different channels involved in molecular ionization, then one must isolate the asymptotic nuclear $E_{N}$ and electronic $\varepsilon_{e l}$ energies that lead to the total energy $E=E_{N}+\varepsilon_{e l}$. To do so, one has to select a particular electronic state and, from there, determine the asymptotic electronic and nuclear energies. We start from the ansatz

$$
\Psi_{i}^{v}(\mathbf{r}, \mathbf{R})=\varphi_{i}(\mathbf{r}, \mathbf{R}) \chi_{i}^{v}(\mathbf{R}),
$$

where $i$ refers to the quantum numbers describing the electronic states, $v$ refers to the quantum numbers associated with the nuclear states, and

$$
\begin{aligned}
\hat{H}_{e l} \varphi_{i}(\mathbf{r}, \mathbf{R}) & =E_{i}(\mathbf{R}) \varphi_{i}(\mathbf{r}, \mathbf{R}), \\
{\left[E_{i}(\mathbf{R})+\hat{T}_{N}\right] \chi_{i}^{v}(\mathbf{R}) } & =W_{i}^{v} \chi_{i}^{v}(\mathbf{R}),
\end{aligned}
$$

which are the well-known eigenequations for the electronic wave function [Eq. (8a)] and the vibrational wave function supported by the potential energy curve $E_{i}(\mathbf{R})$ [Eq. (8b)]. The above equations represent the total molecular wave function within the $\mathrm{BO}$ approximation. In this approximation, due to the mass ratio between the electron and the nuclei, the operator $\hat{T}_{N}$ applied on $\left|\Psi_{i}^{\nu}\right\rangle$ can be approximated by

$$
\hat{T}_{N} \Psi_{i}^{\nu}(\mathbf{r}, \mathbf{R}) \approx \varphi_{i}(\mathbf{r}, \mathbf{R})\left[\hat{T}_{N} \chi_{i}^{\nu}(\mathbf{R})\right],
$$

which implies that the evolution of $\varphi_{i}(\mathbf{r}, \mathbf{R})$ depends smoothly on the nuclear coordinates. For a one-electron diatomic molecule, the latter equation is exact at infinite internuclear distance. By using the latter equation when applying the field-free Hamiltonian onto $\Psi_{i}^{v}$, one gets

$$
\begin{aligned}
\hat{H}_{0} \Psi_{i}^{v}(\mathbf{r}, \mathbf{R}) & =\left(\hat{H}_{e l}+\hat{T}_{N}\right) \varphi_{i}(\mathbf{r}, \mathbf{R}) \chi_{i}^{v}(\mathbf{R}) \\
& =\left[\hat{H}_{e l} \varphi_{i}(\mathbf{r}, \mathbf{R})\right] \chi_{i}^{v}(\mathbf{R})+\varphi_{i}(\mathbf{r}, \mathbf{R})\left[\hat{T}_{N} \chi_{i}^{v}(\mathbf{R})\right] \\
& =W_{i}^{v} \varphi_{i}(\mathbf{r}, \mathbf{R}) \chi_{i}^{v}(\mathbf{R}) .
\end{aligned}
$$

In the case of ionization, Eq. (8a) can be written as $\hat{H}_{e l} \varphi_{i}(\mathbf{r}, \mathbf{R})=\left[E_{i}(\mathbf{R})+\varepsilon_{e l}^{i}\right] \varphi_{i}(\mathbf{r}, \mathbf{R})$, and Eq. $(8 \mathrm{~b})$ as $\left[E_{i}(\mathbf{R})+\right.$ $\left.\varepsilon_{e l}^{i}+\hat{T}_{N}\right] \chi_{i}^{v}(\mathbf{R})=W_{i}^{v} \chi_{i}^{v}(\mathbf{R})$. The term $\varepsilon_{e l}^{i}$ represents the energy of the ionized electron and $E_{i}(\mathbf{R})$ is the potential energy curve of the residual ion. If there is only one such ionization potential (as is usually the case in the SAE), then $E_{i}(\mathbf{R})$ is simply replaced with $E(\mathbf{R})\left(=1 / R\right.$ for $\left.\mathrm{H}_{2}{ }^{+}\right)$. The meanings of the different energies used in these equations are depicted in the schematic given in Fig. 1.

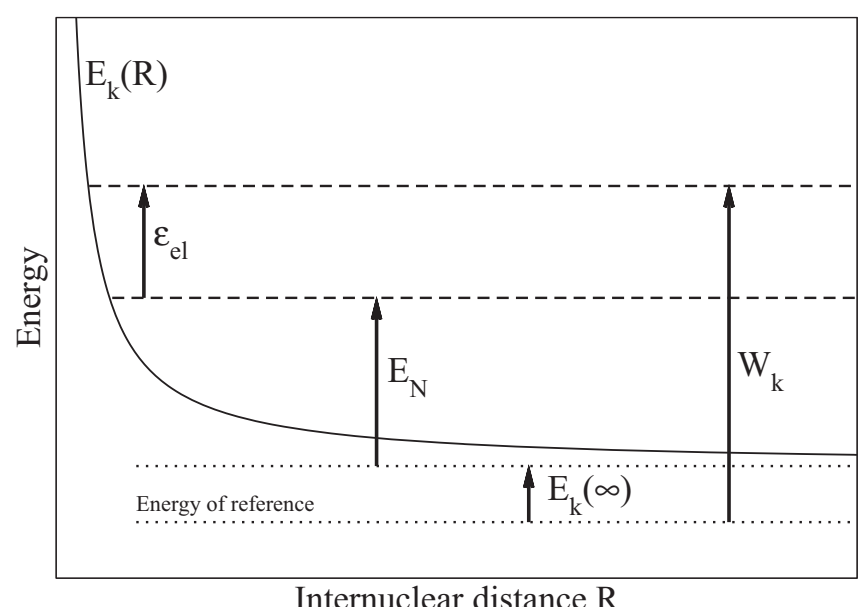

FIG. 1. Schematics of the different quantities used in the ROM analysis. $\varepsilon_{e l}$ is the electron energy in the continuum associated with the ionization potential energy curve $E_{k}(R) . E_{N}$ is the nuclear energy in the asymptotic region. $W_{k}$ is the vibronic energy referred to a given energy [in this example $E_{k}(\infty)$ ], so that $W_{k}=\varepsilon_{e l}+E_{N}+E_{k}(\infty)$. In the case of Coulomb explosion (ionization) of $\mathrm{H}_{2}{ }^{+}, W_{k}=\varepsilon_{e l}+E_{N}$. 
The fact that the wave function can be split into an electronic and a nuclear part allows us to define a resolvent operator for each fragment. The resulting operator is then

$$
\begin{aligned}
\hat{R}\left(E_{N}, \varepsilon_{e l}, k, \delta_{e l}, \delta_{N}, n_{e}, n_{N}\right) & =\frac{\delta_{N}^{n_{N}}}{\left[\left(\hat{T}_{N}+E_{k}(\mathbf{R})\right)-E_{N}-E_{k}(\infty)\right]^{n_{N}}-i \delta_{N}^{n_{N}}} \times \frac{\delta_{e l}^{n_{e}}}{\left[\hat{H}_{e l}-E_{k}(\mathbf{R})-\varepsilon_{e l}\right]^{n_{e}}-i \delta_{e l}^{n_{e}}} \\
& \equiv \hat{R}_{N} \times \hat{R}_{e l},
\end{aligned}
$$

which is a direct product of two resolvent operators associated, respectively, to the electronic energy $\left(\varepsilon_{e l}\right)$ and nuclear energy $\left(E_{N}\right)$ supported by the potential electronic curve $E_{k}(\mathbf{R})$. Here $n_{i}$ is the order of the resolvent operator for fragment $i$ and $\delta_{i}$ the energy resolution for that fragment. The choice of the latter parameters depend on how much resolution one is interested in. In practice, each resolvent operator defined in Eq. (11) is calculated using the Green's operator as defined in Eq. (6). If $E_{k}(\mathbf{R})$ refers to a bound potential curve of the electron, then $\varepsilon_{e l}$ is set to 0 , while for a continuum state, $\varepsilon_{e l}$ refers to the electron energy in the continuum. In the following we give a physical interpretation based on the BO eigenstates, although it must be noted that these states are not calculated in practice.

\section{Interpretation of the ROM}

Most methods used to solve the TDSE involve a space discretization, so that the Hamiltonian spectrum is fully discretized, even for continuum states [43]. Therefore, the integral over continuum states in the wave function can be replaced with a sum in the BO basis of eigenstates,

$$
\begin{aligned}
\Psi(\mathbf{r}, \mathbf{R})= & \sum_{\forall i \text { bound, } \forall v} c_{i}^{v} \varphi_{i}(\mathbf{r}, \mathbf{R}) \chi_{i}^{v}(\mathbf{R}) \\
& +\int d \varepsilon c^{v}(\varepsilon) \varphi_{\varepsilon}(\mathbf{r}, \mathbf{R}) \chi_{\varepsilon}^{v}(\mathbf{R}) \\
\approx & \sum_{\forall i \forall v} c_{i}^{v} \varphi_{i}(\mathbf{r}, \mathbf{R}) \chi_{i}^{v}(\mathbf{R}),
\end{aligned}
$$

where $i$ denotes electronic states and $v$ nuclear (vibrational) states. Applying the resolvent operator on that wave function, and using a given curve $E_{k}(\mathbf{R})$, a nuclear energy $E_{N}$, and an electronic energy $\varepsilon_{e l}$ in the general case where $\varepsilon_{e l} \neq 0$,

$$
\begin{aligned}
\left|\Psi^{\prime}\right\rangle \equiv & \hat{R}|\Psi\rangle=\sum_{\forall i, \forall v} c_{i}^{v} \hat{R}\left|\varphi_{i}(\mathbf{r}, \mathbf{R})\right\rangle\left|\chi_{i}^{\nu}(\mathbf{R})\right\rangle \\
\approx & \sum_{\nu} c_{k}^{v} \frac{\delta_{N}^{n_{N}}}{\left[\left(W_{k}^{v}-\varepsilon_{e l}^{k}\right)-E_{N}-E_{k}(\infty)\right]^{n_{N}}-i \delta_{N}^{n_{N}}} \\
& \times \frac{\delta_{e l}^{n_{e}}}{\left[E_{k}(\mathbf{R})+\varepsilon_{e l}^{k}-E_{k}(\mathbf{R})-\varepsilon_{e l}\right]^{n_{e}}-i \delta_{e l}^{n_{e}}} \\
& \times\left|\varphi_{k}(\mathbf{r}, \mathbf{R})\right\rangle\left|\chi_{k}^{v}(\mathbf{R})\right\rangle \\
= & \sum_{\nu} c_{k}^{v} \frac{\delta_{N}^{n_{N}}}{\left[\left(W_{k}^{v}-\varepsilon_{e l}^{k}\right)-E_{N}-E_{k}(\infty)\right]^{n_{N}}-i \delta_{N}^{n_{N}}} \\
& \times \frac{\delta_{e l}^{n_{e}}}{\left[\varepsilon_{e l}^{k}-\varepsilon_{e l}\right]^{n_{e}}-i \delta_{e l}^{n_{e}}}\left|\varphi_{k}(\mathbf{r}, \mathbf{R})\right\rangle\left|\chi_{k}^{v}(\mathbf{R})\right\rangle,
\end{aligned}
$$

where Eqs. (8) and (11) have been used and we have assumed that the sum is dominated by the term with $j=k$. This is essentially true when $\delta_{e l} \ll\left|E_{j}(\mathbf{R})-E_{k}(\mathbf{R})\right|$, i.e., when the energy difference between the $j$ th and $k$ th electronic curves is bigger than the electronic resolution $\delta_{e l}$. If this relation is not satisfied (which can occur at large distances $R$ in the region close to the ionization threshold), then several kets with approximately the same energy may contribute to the sum.

The modulus square of the transformed wave function is

$$
\begin{aligned}
\left\langle\Psi^{\prime} \mid \Psi^{\prime}\right\rangle= & \sum_{\nu}\left|c_{j}^{\nu}\right|^{2} K\left[W_{k}^{v}-\varepsilon_{e l}^{k}-E_{N}-E_{k}(\infty), \delta_{N}, n_{N}\right] \\
& \times K\left(\varepsilon_{e l}-\varepsilon_{e l}^{k}, \delta_{e l}, n_{e l}\right)
\end{aligned}
$$

where we have used $K(x, q, n)=\frac{q^{2 n}}{x^{2 n}+q^{2 n}}$ and made use of the orthonormality of the BO states. The function $K$ is similar to a window function; i.e., it is close to unity when $x \approx 0$ and close to zero elsewhere. Its width decreases with increasing $n$, so that the quantity $\left\langle\Psi^{\prime} \mid \Psi^{\prime}\right\rangle$ defined by Eq. (15) is small unless $E_{N}=$ $W_{k}^{v}-E_{k}(\infty)-\varepsilon_{e l}^{k}$ and $\varepsilon_{e l}=\varepsilon_{e l}^{k}$, in which case $\left\langle\Psi^{\prime} \mid \Psi^{\prime}\right\rangle=$ $\left|c_{j}^{\nu}\right|^{2}=\left|\left\langle\Psi \mid \varphi_{j} \chi_{j}^{\nu}\right\rangle\right|^{2}$. Therefore, the probability of having a nuclear energy $E_{N}$ and an electronic energy $\varepsilon_{e l}$ supported by a potential curve $E_{k}(\mathbf{R})$ in the wave function $|\Psi\rangle$ is given by

$$
P\left(E_{N}, \varepsilon_{e l}, \delta_{e l}, \delta_{N}, n_{e}, n_{N}\right)=\left\langle\Psi\left|\hat{R}^{\dagger} \hat{R}\right| \Psi\right\rangle,
$$

where we have used the definition of the operator $\hat{R}$ given in Eq. (11). Again, if $E_{k}(\mathbf{R})$ refers to a potential curve for an electron in a bound state, then $\varepsilon_{e l}$ is set to 0 .

The physical quantity that is usually measured is a probability density in energy (and/or angle), while the ROM provides dimensionless probabilities. Therefore, a normalization procedure is needed to properly describe the continuum $\mathrm{BO}$ states [35]. This procedure is explained in the case of the ROM in the next section.

\section{Normalization of the ROM to obtain probability densities}

After the interaction of the molecule with the laser pulse, the final state can either be a bound electronic state, in which case $E_{k}(\mathbf{R})$ defines a curve for a bound electronic orbital or an ionized state. In the first case, the probability is differential in the nuclear energy. In the second (dissociative ionization), the probability is differential both in nuclear and electronic energy.

We specifically pay attention to the latter situation. In this case, Eq. (15) is expressed in terms of continuum functions, which simplifies the analysis of the distribution. Note that while we describe here all the steps to obtain the normalization, in practice only Eqs. (6) and (11) are used. The wave function $|\Psi\rangle$ can be expressed as

$$
|\Psi\rangle=\int d \varepsilon \int_{W_{\varepsilon}>\varepsilon+E(\infty)} d W_{\varepsilon} c\left(\varepsilon, W_{\varepsilon}\right)\left|\varphi_{\varepsilon}\right\rangle\left|\chi_{\varepsilon}^{W_{\varepsilon}}\right\rangle,
$$


where only one of the ionizing potential curve was included. Note that in that case, the coefficient $c\left(\varepsilon, W_{\varepsilon}\right)$ has a dimension; see Eq. (21). Applying the resolvent one gets

$$
\begin{aligned}
\left\langle\Psi\left|\hat{R}^{\dagger} \hat{R}\right| \Psi\right\rangle= & \int d \varepsilon \int_{W_{\varepsilon}>E(\infty)+\varepsilon} d W_{\varepsilon}\left|c\left(\varepsilon, W_{\varepsilon}\right)\right|^{2} \\
& \times K\left(W_{\varepsilon}-W_{\varepsilon_{e l}}, \delta_{N}, n_{N}\right) K\left(\varepsilon-\varepsilon_{e l}, \delta_{e l}, n_{e l}\right) .
\end{aligned}
$$

Assuming that the variation of $c\left(\varepsilon, W_{\varepsilon}\right)$ is much slower than that of $K$, then $\left|c\left(\varepsilon_{e l}, W_{\varepsilon_{e l}}\right)\right|^{2}$ can be taken out of the integral, and Eq. (18) is well approximated by [38]

$$
\begin{aligned}
\left\langle\Psi\left|\hat{R}^{\dagger} \hat{R}\right| \Psi\right\rangle \approx & \left|c\left(\varepsilon_{e l}, W_{\varepsilon_{e l}}\right)\right|^{2} \int d \varepsilon \int_{W_{\varepsilon}>E(\infty)+\varepsilon} d W_{\varepsilon} \\
& \times K\left(W_{\varepsilon}-W_{\varepsilon_{e l}}, \delta_{N}, n_{N}\right) K\left(\varepsilon-\varepsilon_{e l}, \delta_{e l}, n_{e l}\right) \\
\approx & \left|c\left(\varepsilon_{e l}, W_{\varepsilon_{e l}}\right)\right|^{2} \int_{-\infty}^{\infty} d \varepsilon \int_{-\infty}^{\infty} d W_{\varepsilon} \\
& \times K\left(W_{\varepsilon}-W_{\varepsilon_{e l}}, \delta_{N}, n_{N}\right) K\left(\varepsilon-\varepsilon_{e l}, \delta_{e l}, n_{e l}\right) \\
= & \left|c\left(\varepsilon_{e l}, W_{\varepsilon_{e l}}\right)\right|^{2} N\left(\delta_{N}\right) N\left(\delta_{e l}\right),
\end{aligned}
$$

where

$$
N(x)=\frac{\pi}{n} x \csc \frac{\pi}{2 n} .
$$

The probability density is then obtained analogously to Eq. (5), i.e., by dividing by $N\left(\delta_{N}\right)$ and $N\left(\delta_{e l}\right)$ :

$$
\begin{aligned}
\rho\left(\varepsilon_{e l}, W_{\varepsilon_{e l}}\right) & =\frac{\left\langle\Psi\left|\hat{R}^{\dagger} \hat{R}\right| \Psi\right\rangle}{N\left(\delta_{N}\right) N\left(\delta_{e l}\right)}=\left|c\left(\varepsilon_{e l}, W_{\varepsilon_{e l}}\right)\right|^{2} \\
& =\frac{d^{2} P}{d W_{\varepsilon_{e l}} d \varepsilon_{e l}}=\frac{d^{2} P}{d E_{N} d \varepsilon_{e l}} .
\end{aligned}
$$

The latter quantity is actually independent of the choice of the energy resolution $\delta_{N}$ and $\delta_{e l}$. Note that if the potential curve $E_{k}(\mathbf{R})$ is not describing an ionizing state, then the probability density is singly differential, $d P / d E_{N}$, since $\varepsilon_{e l}=0$. Equation(21) defines the link between the probability density and the probability extracted from the ROM in the case of dissociative ionization. If we focus now on dissociative nonionizing channels, then the link between these quantities is

$$
\rho_{k}\left(E_{N}\right)=\frac{\left\langle\Psi\left|\hat{R}_{k}^{\dagger} \hat{R}_{k}\right| \Psi\right\rangle}{N\left(\delta_{N}\right)}=\left|c\left(E_{N}\right)\right|^{2}=\frac{d P_{k}}{d E_{N}},
$$

where $N\left(\delta_{N}\right)$ is given by Eq. (20), and $\hat{R}_{k}$ is defined by

$$
\begin{aligned}
\hat{R}_{k}= & \frac{\delta_{N}^{n_{N}}}{\left[\left(\hat{T}_{N}+E_{k}(\mathbf{R})\right)-E_{N}-E_{k}(\infty)\right]^{n_{N}}-i \delta_{N}^{n_{N}}} \\
& \times \frac{\delta_{e l}^{n_{e}}}{\left(\hat{H}_{e l}-E_{k}(\mathbf{R})\right)^{n_{e}}-i \delta_{e l}^{n_{e}}} .
\end{aligned}
$$

\section{E. Link between the total ROM and the differential ROM}

So far we have detailed the method for extracting the probability density as a function of the total energy of the system (Sec. III A), as well as the differential probability density as a function of both the electronic and nuclear energies
(Sec. III B). The first method involves no approximations, while the latter assumes a separation of the electronic and nuclear degrees of freedom in the extraction of the probability, which is only exact asymptotically. Here we show the link between both approaches by demonstrating how to extract the total ROM probability density from the differential ROM probability density.

We can express the final wave function in the basis of the BO eigenfunctions,

$$
\Psi_{f}(\mathbf{r}, \mathbf{R})=\sum_{i, v} c_{i}^{v} \varphi_{i}(\mathbf{r}, \mathbf{R}) \chi_{i}^{v}(\mathbf{R}),
$$

where once again the discrete sum for continuum states is due to the discretization of the energy spectrum in the grid. We define the correlation function as

$$
\begin{aligned}
& \sigma_{E_{\text {tot }}, T}=\frac{1}{2 \pi} \operatorname{Re}\left[\int_{-T_{\text {prop }}}^{T_{\text {prop }}} d \tau e^{-i E_{\text {tot }} \tau}\left\langle\Psi(\tau) \mid \Psi_{f}\right\rangle\right] \\
& =\frac{1}{2 \pi} \operatorname{Re}\left[\int_{-T_{\text {prop }}}^{T_{\text {prop }}} d \tau e^{-i E_{\mathrm{tot}} \tau}\left\langle\Psi_{f}\left|U^{\dagger}(\tau)\right| \Psi_{f}\right\rangle\right] \\
& =\frac{1}{2 \pi} \operatorname{Re}\left[\int_{-T_{\text {prop }}}^{T_{\text {prop }}} d \tau e^{-i E_{\mathrm{tot}} \tau}\right. \\
& \left.\times \sum_{i, v} c_{i}^{\mathcal{v} *}\left|\varphi_{i} \chi_{i}^{\nu}\right| e^{i\left[\varepsilon_{e l}+E_{N}+E_{\mathrm{ion}}(\infty)\right] \tau}\left|\Psi_{f}\right\rangle\right],
\end{aligned}
$$

where $T_{\text {prop }}$ is the propagation time which is larger than the pulse duration. By using Eq. (24) and the orthonormality of the $\mathrm{BO}$ eigenfunctions, one obtains

$$
\sigma_{E_{\mathrm{tot}}, T}=\frac{1}{\pi} \sum_{i, v}\left|c_{i}^{\nu}\right|^{2} \frac{\sin \left\{\left[E_{\mathrm{tot}}-\varepsilon_{e l}-E_{N}-E_{\mathrm{ion}}(\infty)\right] T_{\mathrm{prop}}\right\}}{E_{\mathrm{tot}}-\varepsilon_{e l}-E_{N}-E_{\mathrm{ion}}(\infty)} .
$$

For $T_{\text {prop }} \rightarrow \infty$ and using $\lim _{T_{\text {prop }} \rightarrow \infty} \frac{\sin (x T)}{x}=\pi \delta(x)$, we get

$$
\begin{aligned}
\frac{d P_{\mathrm{ion}}}{d E_{\mathrm{tot}}} & =\sigma_{E_{\mathrm{tot}}, T} \\
& =\sum_{i, \nu}\left|c_{i}^{\nu}\right|^{2} \delta\left(E_{\mathrm{tot}}-\varepsilon_{e l}-E_{N}-E_{\mathrm{ion}}(\infty)\right),
\end{aligned}
$$

where the discrete summation can be replaced with a double integral, giving the formal expression

$$
\begin{aligned}
\frac{d P_{\text {ion }}}{d E_{\mathrm{tot}}}= & \iint d \varepsilon_{e l} d E_{N} \frac{d^{2} P_{\text {ion }}}{d \varepsilon d E_{N}} \\
& \times \delta\left(E_{\mathrm{tot}}-\varepsilon_{e l}-E_{N}-E_{\text {ion }}(\infty)\right) .
\end{aligned}
$$

The previous definition holds for dissociative ionization, for which $E_{\mathrm{ion}}(\infty)=0$ is usually defined. Analogously, for bound states $\left(\varepsilon_{e l}=0\right)$, the probability density as a function of the total energy for a given channel $q$ is

$$
\frac{d P_{q}}{d E_{\mathrm{tot}}}=\int d E_{N} \frac{d P_{q}}{d E_{N}} \delta\left(E_{\mathrm{tot}}-E_{N}-E_{q}(\infty)\right) .
$$


Therefore, in differential ROM, Eqs. (28) and (29) correspond to the probability of having a total energy $E_{\text {tot }}=\varepsilon_{e l}+E_{N}$, for any given values of $\varepsilon_{e l}$ and $E_{N}$ [assuming $E_{\text {ion }}(\infty)=0$ ].

The probability density as a function of the total energy is the incoherent sum of the different contributions previously described,

$$
\frac{d P}{d E_{\mathrm{tot}}}=\frac{d P_{\mathrm{ion}}}{d E_{\mathrm{tot}}}+\sum_{\forall q} \frac{d P_{q}}{d E_{\mathrm{tot}}},
$$

where the sum in $q$ runs over all nonionizing dissociative channels. Equation (30) links the total ROM [Eq. (5)], where no approximations are performed, to the differential ROM, where the separation of electronic and nuclear coordinates is invoked while obtaining the spectrum. For the calculation of the differential ROM the final wave function has been projected onto its gerade or ungerade contributions, depending on the symmetry of the chosen bound potential electronic curve. This procedure minimizes problems that could occur due to the degeneracy of different potential curves, particularly at large internuclear distances.

At this point, it is important to stress that the BO approximation is no longer an approximation when the two atomic centers are infinitely separated, as, e.g., in the dissociative ionization channels considered in this work. Therefore, our factorization of the resolvent operator into a purely electronic part and a purely nuclear part [see Eq. (11)] becomes exact when $T_{\text {prop }} \rightarrow \infty$. So, in practice, if propagation of the TDSE is carried out up to sufficiently long times, application of the resolvent operator defined in Eq. (11) should provide the exact result. To check this last point in the present calculations, we have evaluated dissociative ionization probabilities by applying the resolvent operator at different times after the end of the pulse [i.e., the TDSE is propagated up to different values of $T_{\text {prop }}\left(T_{\text {prop }}>T\right)$, where the resolvent operator is applied]. The results are shown in Fig. 10 of Appendix B. As can be seen, by increasing the propagation time well beyond the end of the pulse, the calculated probabilities do not change, thus showing that we have reached the limit for which the use of the $\mathrm{BO}$ approximation to extract the ionization yield becomes an exact procedure.

\section{RESULTS}

In this section we present results for electron-, nuclear-, and correlated electron- and nuclear-kinetic energy spectra for $\mathrm{H}_{2}{ }^{+}$ionization and dissociation by ultrashort laser pulses. We study the validity of the differential ROM, as well as its comparison with the total ROM described in the previous section. In order to do that, we consider strong $\left(>10^{13} \mathrm{~W} / \mathrm{cm}^{2}\right)$ ultrashort (few femtoseconds) laser pulses with frequencies ranging from XUV to IR. It must be noted that the ROM can be applied in a wide range of intensities (in principle, up to the validity of the nonrelativistic TDSE equation) and frequencies, as well as pulse durations, from attosecond laser pulses to longer pulses. The upper limitation to the pulse duration is that the wave function must be fully contained inside the grid when the ROM analysis is performed. Therefore, for longer pulses, bigger grids are needed.

\section{A. Correlated photoelectron- and proton-kinetic energy spectra}

We first focus on the differential ROM, which provides correlated photoelectron- and nuclear-kinetic energy spectra (CKE). For all calculations in this section, the values $n_{e l}=$ $n_{N}=2$ and $\delta_{e l}=\delta_{N}=0.004$ in Eq. (11) are used. We have checked that by increasing the values of $\delta_{e l}$ and $\delta_{N}$ by $50 \%$, the results barely change (see Appendix B). The ROM analysis is always performed right after the end of the laser pulse, at the time $T$. In all cases, the initial state was the ground rovibrational state of the $1 s \sigma_{g}{ }^{1}$ electronic state of $\mathrm{H}_{2}{ }^{+}$, denoted $\Psi_{0}^{\nu=0}$. Since we are interested in the part of the wave function that is affected by the laser field, we have removed the initial state from the final wave function before performing the analysis. In this way we remove a possible contamination by the initial state in the calculated doubly differential ionization probabilities.

\section{Few-photon absorption}

The simplest case corresponds to the absorption of one or two photons with an ultrashort XUV laser pulse. Results for a central frequency $\Omega=1.37$ a.u., a total pulse duration of $16 \mathrm{fs}$, and peak intensity $10^{14} \mathrm{~W} / \mathrm{cm}^{2}$ are presented in Fig. 2. These parameters correspond to a purely multiphotonic regime since the Keldysh parameter is $\gamma=38 \gg 1$, where $\gamma=\sqrt{I_{p} / 2 U_{p}}$, with $I_{p}$ the ionization potential at the equilibrium distance $R_{e}=1.9$ a.u., and $U_{p}=I / 4 \Omega^{2}$ the ponderomotive energy.

The BO potential energy curves of the $\mathrm{H}_{2}{ }^{+}$molecule are shown in Fig. 2(a), where the Franck-Condon region lies between the vertical dashed lines. The corresponding CKE spectrum is shown in Fig. 2(b), where the ionization probability is plotted (in log scale) as a function of the electronand nuclear-kinetic energy. We expect to observe energy conservation lines, which obey $N \omega \simeq \varepsilon_{e l}+E_{N}+D_{2 H^{+}}$, where $D_{2 H^{+}}=-E_{0}=0.597$ a.u. is the threshold energy required to produce two protons at infinite internuclear distance, and $E_{0}$ is the energy of the initial state. Therefore, in a $2 \mathrm{D}\left(E_{N}, \varepsilon_{e l}\right)$ plot they correspond to lines with slope -1 . Two of these lines are visible in the figure, corresponding to one- and two-photon absorption $(N=1,2)$. The one-photon absorption line exhibits a minimum at a nuclear energy around 0.56 a.u., which corresponds to an internuclear distance of $R \sim 1.78$ a.u. in the Coulomb explosion curve $1 / R$ [green line in Fig. 2(a)]. This minimum is clearly visible in the integrated nuclear-kinetic energy (NKE) distribution shown in Fig. 2(b), top panel.

The origin of such a minimum in the CKE spectrum can be understood if we resort to one-dimensional calculations in which the internuclear distance is fixed. The corresponding ionization probability, which has been obtained by using the same soft-core potential as in the $(1+1) \mathrm{D}$ calculations, is shown in Fig. 2(c) as a function of the internuclear distance.

\footnotetext{
${ }^{1}$ It has to be noted that the calculations have been performed using a 1D grid for the electronic coordinates, so that no angular momentum can be defined. Nonetheless, the parameters used in $\hat{V}_{e N}$ are such that the potential energy curves of the ground electronic state and the first excited electronic state are close to those of the real 3D case. That is the reason why, for the sake of clarity, we keep the 3D terminology throughout the paper.
} 

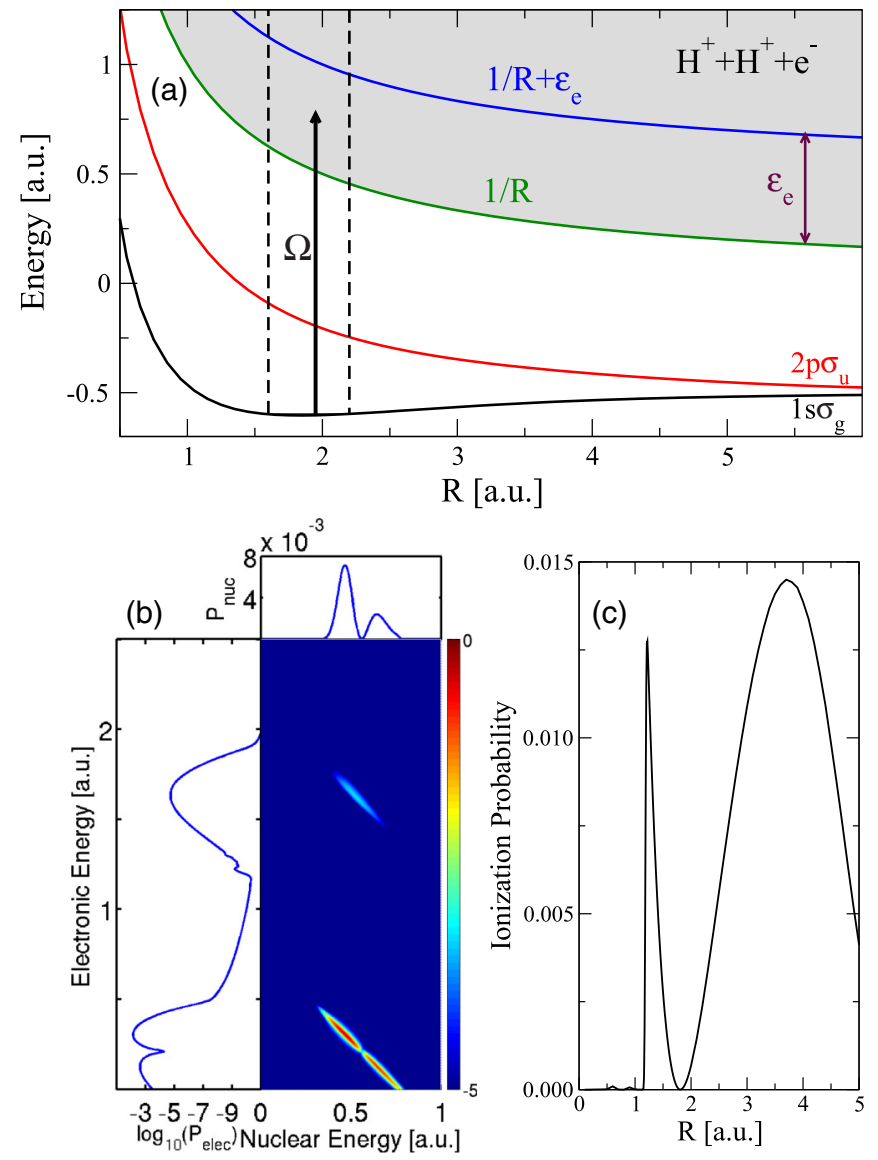

FIG. 2. (Color online) (a) Born-Oppenheimer potential energy curves for the $\mathrm{H}_{2}{ }^{+}$molecule. The black arrow represents a vertical transition from the $\mathrm{H}_{2}{ }^{+}$ground state to the ionization channel with a photon energy $\Omega=1.37$ a.u. The dashed lines represent the limits of the Franck-Condon region. (b) CKE for a pulse with $\Omega=1.37$ a.u., total duration $16 \mathrm{fs}$ and $I=10^{14} \mathrm{~W} / \mathrm{cm}^{2}$. The corresponding projections (singly differential probabilities) in electronic energy $\left(P_{\text {elec }}\right)$ and nuclear energy $\left(P_{\text {nuc }}\right)$ are shown on the left and on top of the figure. (c) Ionization probability as a function of the internuclear distance $R$ using the same soft-core potential as in the $(1+1) \mathrm{D}$ calculations.

This ionization probability exhibits a pronounced minimum at $R=1.8$ a.u., which explains the depletion observed in the CKE spectrum. A similar depletion has been observed in $\mathrm{H}_{2}{ }^{+}$ionization probabilities resulting from full dimensional calculations [44]. As in the present case, this is due to a minimum in the dipole-coupling matrix element connecting the ground and the final continuum state of $\mathrm{H}_{2}{ }^{+}$.

To get further insight on the origin of this minimum, we have performed model calculations in the framework of first-order perturbation theory. In these calculations, we have used the representation of the initial and final states proposed by Cohen and Fano [45]: The final state is described by a plane wave and the initial state by a linear combination of $1 s$ orbitals corresponding to the actual nuclear charge $Z=1$, but also to an $R$-dependent effective charge chosen to reproduce the exact ground-state potential energy curve. The ionization probability is obtained by weighting the square of the corresponding dipole matrix element with the ground-state nuclear probability

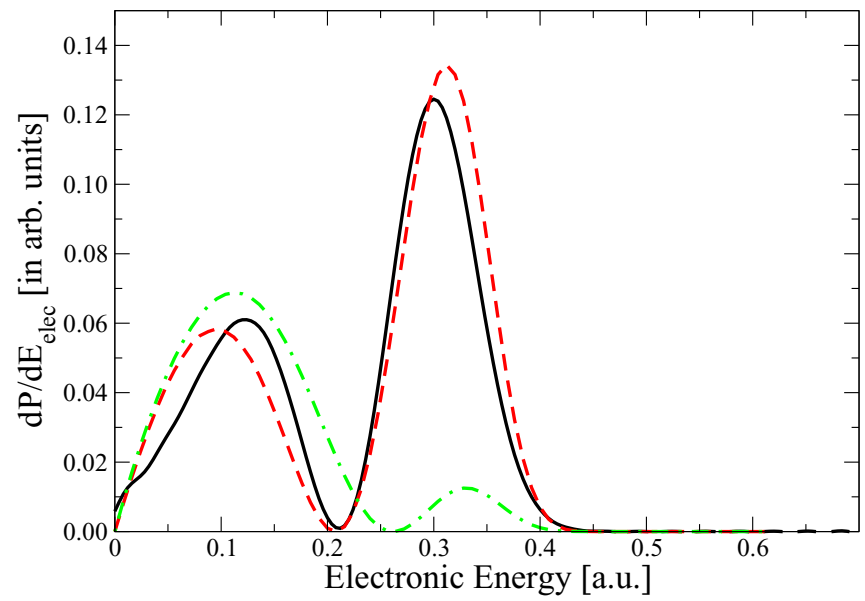

FIG. 3. (Color online) Ionization probability as a function electron energy. Comparison between the results of the full calculations shown in Fig. 2 (black solid curve) and those of the first-order perturbative model described in text with $Z=1$ (green dash-dotted curve) and effective charge (red dashed curve).

density (reflection approximation [46]). A comparison with the results of the TDSE calculations (see Fig. 3) shows that the model with effective charges catches the essential features of the full calculation. Hence, the minimum in the ionization probability can unambiguously be attributed to the interference resulting from the coherent electron emission from the two molecular centers.

\section{Resonant transition}

The differential ROM makes it possible to extract the contributions to the energy spectrum arising from different electronic states of the molecule. To illustrate this we choose a laser pulse with the same duration and intensity as before, but whose energy is resonant to a particular electronic transition, $1 s \sigma_{g} \rightarrow 2 p \sigma_{u}$, instead of leading directly to ionization. At the internuclear equilibrium distance, the energy difference between those states is $E_{2 p \sigma_{u}}-E_{1 s \sigma_{g}} \approx 0.4$ a.u. The seventh harmonic of a $800-\mathrm{nm}$ pulse $(\Omega=0.398$ a.u.) matches well that energy difference. As shown in Fig. 4(a), the final kinetic energy of the nuclei in this situation is expected to be $T_{N} \sim 0.29$ a.u. The NKE spectrum for the $2 p \sigma_{u}$ state calculated with the differential ROM, shown in Fig. 4(c), confirms this prediction. However, in this case we can also have ionization through absorption of three or more photons, via resonance-enhanced multiphoton ionization (REMPI). This can be seen in the CKE spectrum shown in Fig. 4(b), where several lines of energy conservation can be observed.

The integrated EKE spectrum [Fig. 4(b), left panel] is more complex than in the UV case shown in Fig. 2. The maxima corresponding to multiphoton absorption $(N \leqslant 3)$ show a splitting of the peaks due to the Autler-Townes effect and the nuclear motion [47]. In the case of atomic resonant ionization, this splitting can be interpreted in terms of dressed states induced by the field [48]: Each state splits in two with an energy separation equal to the Rabi frequency $\Omega_{R}=\mu E$, where $\mu$ is the dipole matrix element between the resonantly coupled states and $E$ is the maximum amplitude 

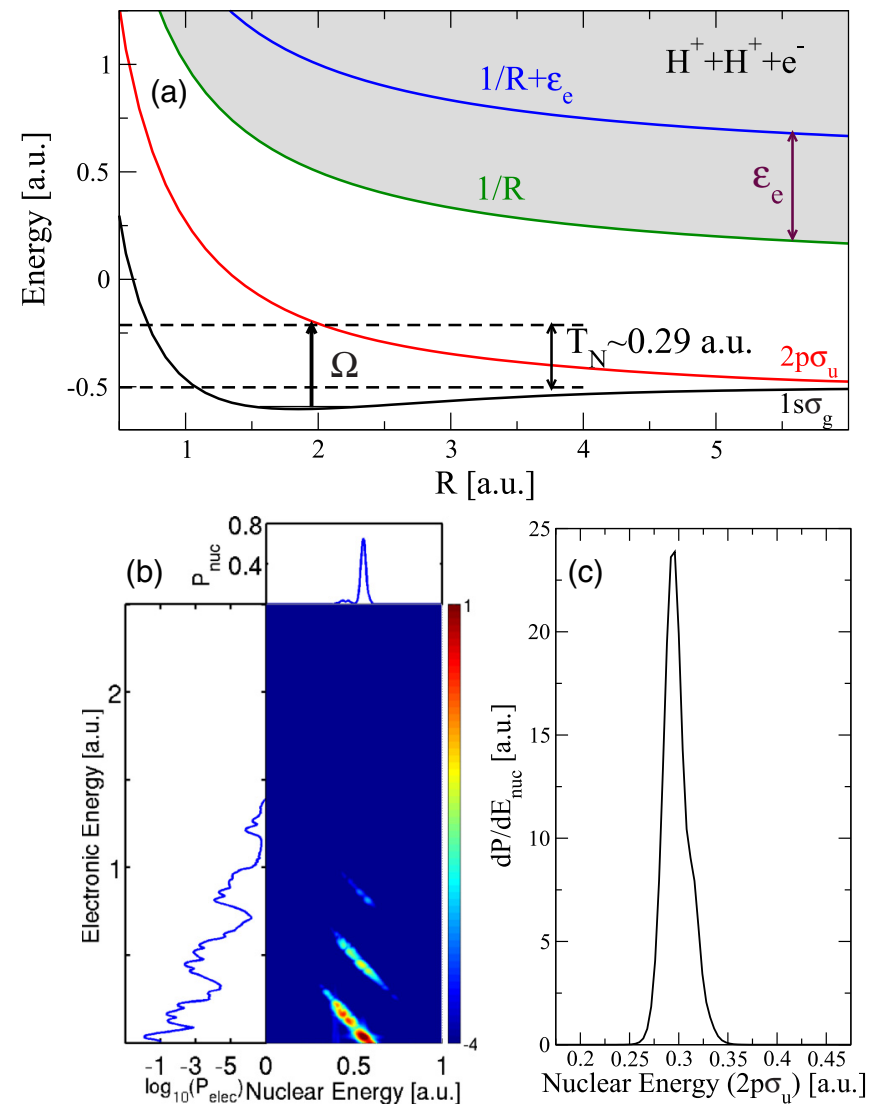

FIG. 4. (Color online) (a) Born-Oppenheimer curves of the $\mathrm{H}_{2}{ }^{+}$ molecule. The black arrow represents a vertical transition from the $\mathrm{H}_{2}{ }^{+}$ground state to the first excited state $\left(1 s \sigma_{g} \rightarrow 2 p \sigma_{u}\right)$ with a photon energy $\Omega=0.398$ a.u. (b) CKE for a pulse with $T=16 \mathrm{fs}$ and $I=10^{14} \mathrm{~W} / \mathrm{cm}^{2}$. The corresponding projections (singly differential probabilities) in electronic energy $\left(P_{\text {elec }}\right)$ and nuclear energy $\left(P_{\text {nuc }}\right)$ are shown on the left and on top of each panel. (c) NKE spectrum of the $2 p \sigma_{u}$ electronic state.

of the electric field. The molecular case is not that simple. For the photon energy used here, $\Omega=0.398$ a.u., the two lowest electronic states are resonantly coupled; see Fig. 5. The dressed $2 p \sigma_{u}$ curve (shifted by $-\Omega$ ) splits into two different curves, so that the energy difference between them is $\Omega_{R}$. This energy difference depends on the internuclear distance because the dipole matrix element $\mu(R)=\left.\left\langle 2 p \sigma_{u}|z| 1 s \sigma_{g}\right\rangle\right|_{R}$ does. An order of magnitude of $\Omega_{R}$ can be obtained by taking the value of $\mu$ at the equilibrium distance $R_{0}=1.9$ a.u., which is $\mu\left(R_{0}\right)=1.084$ a.u., thus leading to $\Omega_{R}=0.058$ a.u. for $10^{14} \mathrm{~W} / \mathrm{cm}^{2}$. This value of $\Omega_{R}$ is compatible with the observed splitting. Now the nuclear motion further complicates this picture. When the nuclear wave packet is centered around $R \sim R_{1}$, the electron is preferentially emitted from the lower curve and, therefore, has smaller kinetic energy than when the nuclear wave packet is centered around $R \sim R_{2}$, since in the latter case the electron is preferentially emitted from the upper curve [Fig. 5(a)]. The energy difference between both channels projected onto the ionization potential curve is $\Delta E \sim 1 / R_{1}-1 / R_{2}=0.059$ a.u., which is very similar to $\Omega_{R}$. As a consequence of this, additional structures due to the different kinetic energies that the electron can acquire as
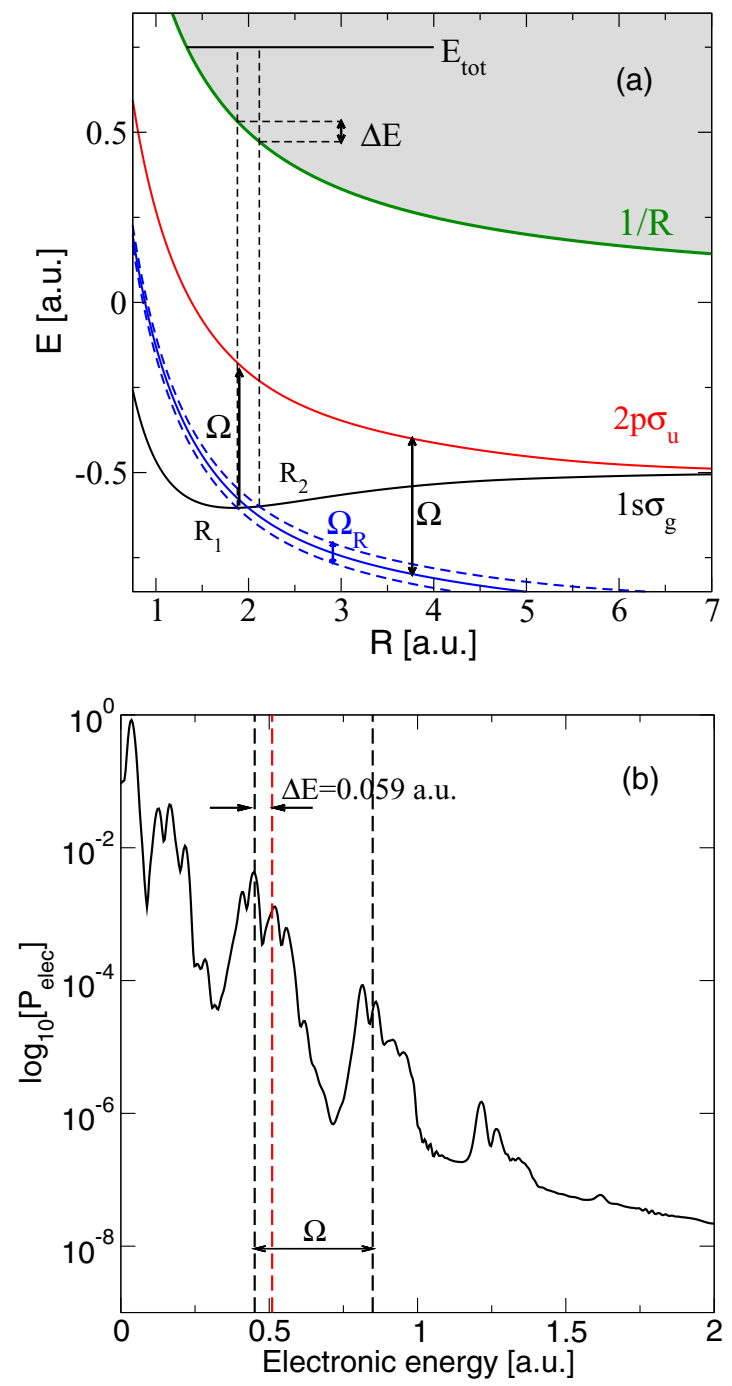

FIG. 5. (Color online) (a) Potential energy curves corresponding to ionization $(1 / R), 2 p \sigma_{u}$ and $1 s \sigma_{g}$ as a function of the internuclear distance. The dressed states of the $2 p \sigma_{u}$ curve are shifted by $\frac{\Omega_{R}}{2}$ (upward and downward). The diabatic coupling of these states is represented by the vertical dashed lines. (b) EKE shown in Fig. 4(b).

the nuclear wave packet moves are expected to appear in an energy interval similar to that dictated by the Autler-Townes splitting.

\section{Transition regime between multiphoton ionization and tunneling ionization}

One of the most demanding situations for extracting energy spectra corresponds to intense low-frequency fields. The absorption of a large number of photons may lead to electrons with a high angular momentum, which represents a challenge for spectral methods, in which the basis set must include states for a large number of angular momenta. The storage of all the dipole matrix elements required in the latter method is a real bottleneck that is easily overcome in grid methods. With these fields, both multiphoton absorption and tunneling are possible. In Appendix $\mathrm{C}$ a quantitative comparison to the data previously obtained by Madsen et al. [19] in the multiphoton region is 

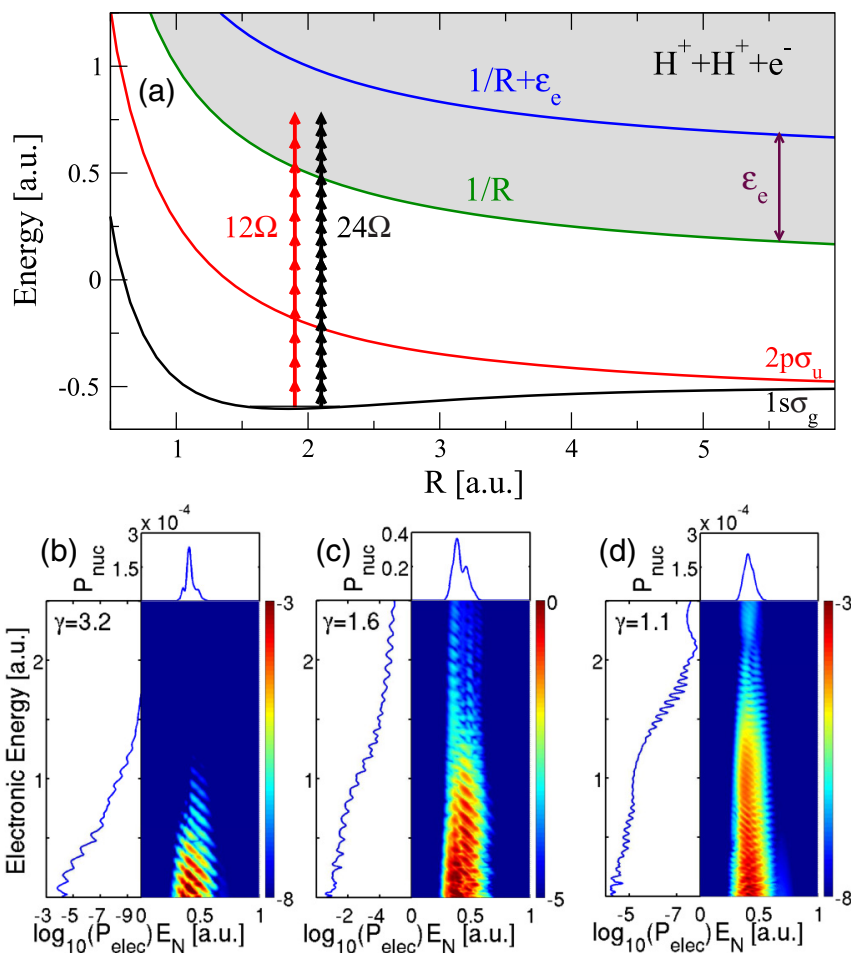

FIG. 6. (Color online) (a) Born-Oppenheimer curves of the $\mathrm{H}_{2}{ }^{+}$ molecule. The short (long) arrows represent a vertical transition from the $\mathrm{H}_{2}{ }^{+}$ground state to the ionization channel by absorbing 24 (12) photons of a $800-\mathrm{nm}(400-\mathrm{nm})$ pulse. The bottom figures show the CKE for the following pulses: (b) $400 \mathrm{~nm}$ and $I=10^{14} \mathrm{~W} / \mathrm{cm}^{2}$, (c) $400 \mathrm{~nm}$ and $I=4 \times 10^{14} \mathrm{~W} / \mathrm{cm}^{2}$, and (d) $800 \mathrm{~nm}$ and $I=$ $2 \times 10^{14} \mathrm{~W} / \mathrm{cm}^{2}$. The pulse duration is $T=16 \mathrm{fs}$ in all cases. The corresponding projections (singly differential probabilities) in electronic energy $\left(P_{\text {elec }}\right)$ and nuclear energy $\left(P_{\text {nuc }}\right)$ are shown on the left and on top of each panel.

presented. The comparison shows a good agreement (up to a global normalization factor). We have additionally checked that the total ionization probability obtained with our method is almost identical to those obtained with other methods such as the virtual detector method [49]. The differential ROM will allow us to track the transition from the multiphoton regime $(\gamma>1)$ to the tunneling regime $(\gamma \leqslant 1)$ [16]. This is shown in Fig. 6 for three pulses: $400 \mathrm{~nm}$ and $10^{14} \mathrm{~W} / \mathrm{cm}^{2}$ (corresponding to $\gamma=3.2$ ), $400 \mathrm{~nm}$ and $4 \times 10^{14} \mathrm{~W} / \mathrm{cm}^{2}$ $(\gamma=1.6)$, and $800 \mathrm{~nm}$ and $2 \times 10^{14} \mathrm{~W} / \mathrm{cm}^{2}(\gamma=1.1)$, all with a total duration of $T=16 \mathrm{fs}$.

In the purely multiphoton case [Fig. 6(a)] we observe energy conservation lines, which indicates that the excess energy is shared between the nuclear and electronic degrees of freedom. In the tunneling case [Fig. 6(c)] we observe horizontal lines at low EKE, approximately separated by $\Omega$, which are independent of the nuclear-kinetic energy. This indicates that the energy taken by the electrons from the laser field is not shared with the nuclei. As shown in Ref. [16], this is due to electrons resulting from tunnel ionization, where the molecular character is partly lost. The intermediate situation [Fig. 6(b)] is a transition between both regimes, with a complex pattern at low electron energies.

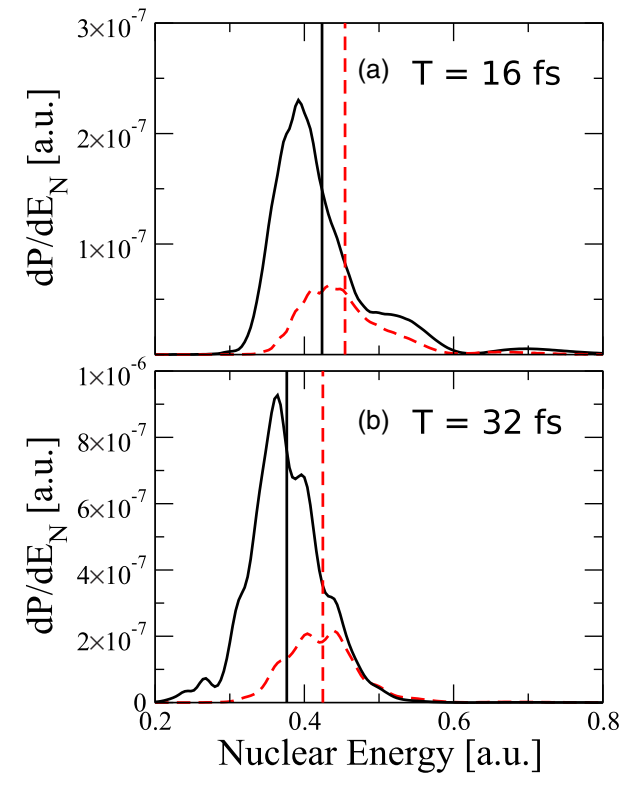

FIG. 7. (Color online) NKE in the ionization channel for $\mathrm{H}_{2}{ }^{+}$ (black solid lines) and $\mathrm{D}_{2}{ }^{+}$(red dashed lines) for a laser pulse of $800 \mathrm{~nm}$ and $10^{14} \mathrm{~W} / \mathrm{cm}^{2}$, with total duration $T=16 \mathrm{fs}$ (a) and $T=32$ fs (b). The vertical lines show the value of $\left\langle E_{N}\right\rangle$ for each case.

\section{B. Isotopic effects}

The nuclear motion has an important role in the shape of the correlated photoionization spectra [16]. Therefore, we expect to find differences when the mass of the molecule changes. This can be tested by using different isotopes of the $\mathrm{H}_{2}{ }^{+}$ molecule. In Fig. 7 we show results for $\mathrm{H}_{2}{ }^{+}(\mu=918.0$ a.u., black lines) and $\mathrm{D}_{2}{ }^{+}(\mu=1835.2$ a.u., red lines $)$ for two different pulse durations: $T=16 \mathrm{fs}$ (a) and $T=32 \mathrm{fs}$ (b). For both pulse durations, the ionization probability is higher for $\mathrm{H}_{2}{ }^{+}$than for $\mathrm{D}_{2}{ }^{+}$. This is due to the fact that when the nuclei move faster, they access higher internuclear distances, for which the number of photons that is necessary to ionize the molecule is smaller.

The average value of the nuclear energy for each case, $\left\langle E_{N}\right\rangle=\int d E_{N} \frac{d P}{d E_{N}} E_{N}$, is shown by vertical lines. We observe that the NKE distribution is shifted towards lower energy values in $\mathrm{H}_{2}{ }^{+}$, which corresponds to higher internuclear distances. This is also due to the faster nuclear motion in $\mathrm{H}_{2}{ }^{+}$. When the pulse is longer [Fig. 7(b)], the nuclei have time to reach even larger internuclear distances, which implies a shift towards lower NKE values for both isotopes.

\section{Total ROM vs differential ROM}

Finally, we discuss the results obtained with the total ROM in comparison to those obtained by integrating the differential ROM. In the latter case, the total probability density for each total energy $E_{\text {tot }}$ results from integration over all possible energy pairs $\left\{\epsilon_{e l}, E_{N}\right\}$ such that $E_{\text {tot }}=\epsilon_{e l}+E_{N}$. To compare the spectra, we have considered the first 15 bound curves of the $\mathrm{H}_{2}{ }^{+}$molecule, as well as all positive electron energies up to 3 a.u. To distinguish the contribution to the total spectrum that comes from ionization, we also calculate the 

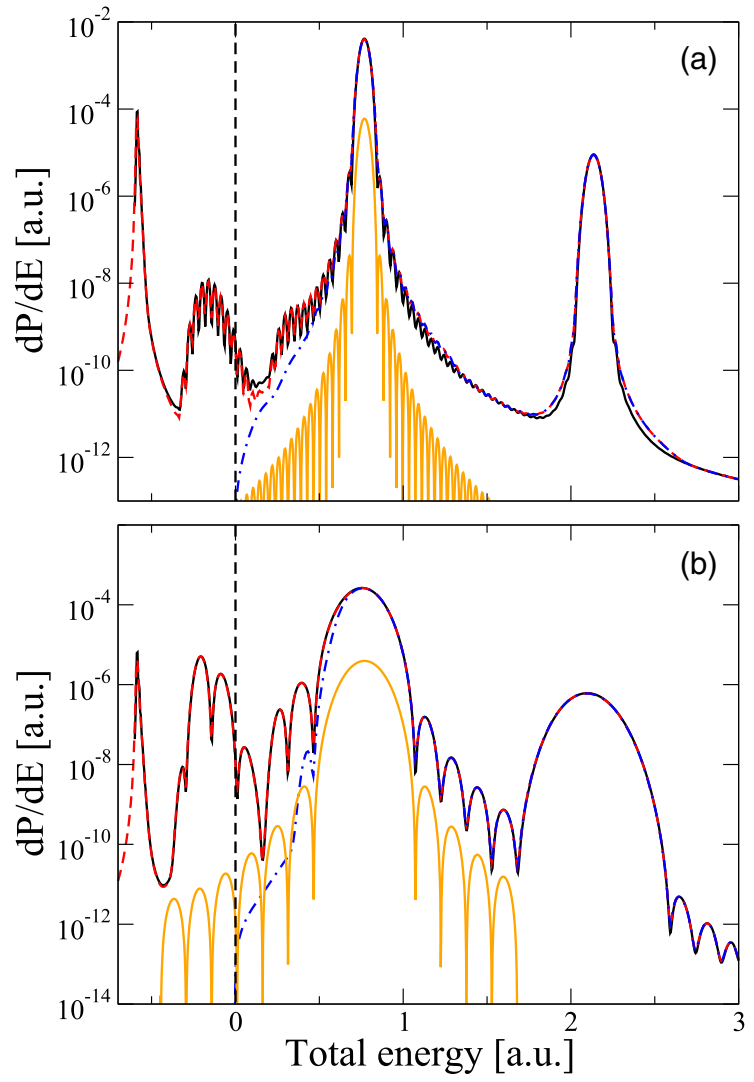

FIG. 8. (Color online) Total density probability calculated by using the total ROM (black solid line) using $n=2$ and $\delta=0.004$ a.u. or by integrating the differential ROM, either including all channels (red dashed line) or only the ionization channel (blue dash-dotted line), for an XUV pulse with $\Omega=1.37$ a.u. and $I=10^{14} \mathrm{~W} / \mathrm{cm}^{2}$. The bottom orange line represents the Fourier transform of the pulse, $|E(\omega)|^{2}$, which has been shifted downward for clarity. Total durations: $T=4 \mathrm{fs}$ (top) and $1 \mathrm{fs}$ (bottom).

ROM spectrum due only to the ionization channel $\left[\frac{d P_{\text {ion }}}{d E_{\text {tot }}}\right.$ in Eq. (30)].

In Fig. 8 we consider the same configuration as in Fig. 2, $\Omega=1.37$ a.u. and $10^{14} \mathrm{~W} / \mathrm{cm}^{2}$, but with total pulse durations of $4 \mathrm{fs}$ (a) and $1 \mathrm{fs}$ (b), corresponding to 36 and 9 optical cycles, respectively. In both cases, the agreement between the results obtained by using the total ROM (black) and those obtained by integrating the differential ROM (red) is excellent for high positive energies. In Fig. 8(a) we observe four main peaks. The leftmost peak, just above the $1 s \sigma_{g}(v=0)$ energy ( -0.597 a.u.), corresponds to excited vibrational states of the ground electronic state, $\Psi_{0}$ (remember that $\Psi_{0}^{\nu=0}$ was removed from the final wave function before performing the ROM analysis). These states are populated via absorption and emission of a photon: $\Psi_{0}^{\nu=0} \stackrel{+\omega}{\longrightarrow} \Psi^{\prime} \stackrel{-\omega^{\prime}}{\longrightarrow} \Psi_{0}^{\nu>0}$, with $\omega^{\prime} \lesssim \omega$, corresponding to the population of vibrational states by Raman processes. Since the laser pulse is short, its spectral bandwidth is wide enough to host photons with energies different enough to allow such transitions. The second peak is located around the $2 p \sigma_{u}$ energy at the equilibrium internuclear distance $R_{0}=1.9$ a.u. ( -0.183 a.u.). The remaining two peaks, at positive energies, correspond to ionization via one- and two-photon absorption from the initial state. The Fourier transform of the laser field is shown in orange in Fig. 8. The small lobes in its spectrum, due to aliasing, are transferred to the molecular spectrum. We note that the population of the $2 p \sigma_{u}$ state is due to one-photon absorption from the $1 s \sigma_{g}$ state with low-energy photons, which correspond to the left energy tail of the pulse.

The first conclusion is that in the regions where the population is high, around these four peaks, the comparison between differential ROM (red) and total ROM (black) is very good. In the regions between peaks there are tails, whose widths are determined by the choice of $\delta$ in the ROM analysis. As shown here, a correct choice of $\delta$ in both the differential ROM and the total ROM provides very similar spectra. When the pulse is shorter [Fig. 8(b)], the energy bandwidth of the pulse is larger. In this situation, the $2 p \sigma_{u}$ state and in general the whole spectrum has a significant population, and the differential and total ROM spectra are indistinguishable.

The contribution from ionizing states is shown in blue in Fig. 8. Ionization takes place whenever an electron is released, and this can happen even for $E_{\mathrm{tot}} \approx 0$ for very high internuclear distances. However, most ionization takes place within the Franck-Condon region. The right limit to that region is located around the outer classical turning point at $R=2.2$ a.u., which corresponds to $1 / R=0.45$ a.u. in the Coulomb explosion curve. For that total energy, ionization becomes the dominant channel, as can be observed in Figs. 8 and 9.

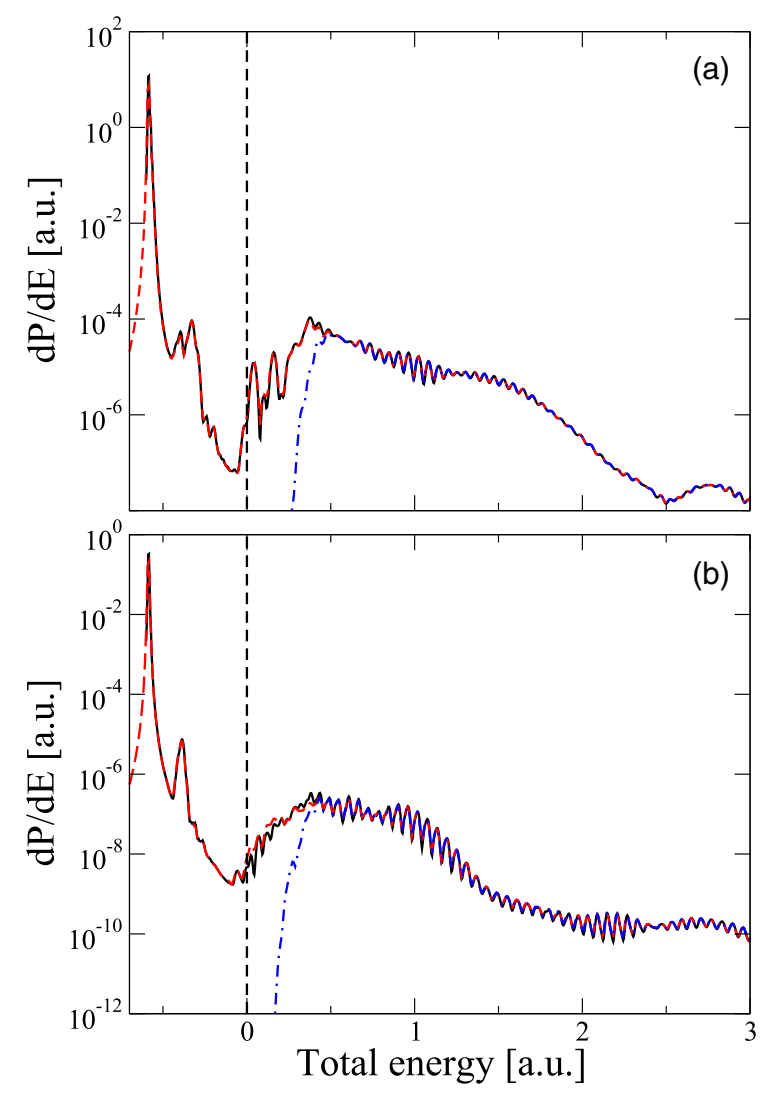

FIG. 9. (Color online) Same as in Fig. 8 for a $800-\mathrm{nm}$ pulse with $T=16$ fs and $I=2 \times 10^{14} \mathrm{~W} / \mathrm{cm}^{2}$ (top) and $T=32 \mathrm{fs}$ and $I=10^{14} \mathrm{~W} / \mathrm{cm}^{2}$ (bottom). 
In Fig. 9(a) we show the multiphoton absorption spectrum of an 800-nm pulse with total pulse duration $T=16$ fs and $I=2 \times 10^{14} \mathrm{~W} / \mathrm{cm}^{2}$, obtained with the differential ROM (red solid line) and total ROM (black dashed line). The ionization channel is shown again in a blue dashdotted line. In the ionization region, the spectrum shows a typical ATI shape, with multiple peaks separated by the photon energy $\Omega=0.057$ a.u. The differential ROM and total ROM calculations are indistinguishable in this region. In the nonionizing region ( $E_{\mathrm{tot}}<0.45$ a.u.) the comparison is still excellent.

In Fig. 9(b) we show results for a longer pulse, with total duration $32 \mathrm{fs}$, and intensity $10^{14} \mathrm{~W} / \mathrm{cm}^{2}$. Here the peaks are narrower due to the smaller spectral width of the pulse. Both methods are still indistinguishable in the ionization region and at negative energies. Small differences can be seen in the region just above the ionization threshold, where ejected electrons are very slow and Rydberg states are expected to play some role. At first sight, one might be tempted to attribute these differences to nonadiabatic couplings between Rydberg states (whose potential energy curves lie just below that of the ionization threshold and run almost parallel to it) and the ionization continuum [50]. Such nonadiabatic effects might not be accounted for by the differential ROM when applied just at the end of the pulse. To check if this is the case, we have performed the ROM analysis at different times after the end of the pulse (see Fig. 11 in Appendix B). One can see that the total energy spectrum resulting from the differential ROM is already converged at the end of the pulse. These results point out that the small differences observed near the ionization threshold (between 0 and 0.5 a.u.) are not due to nonadiabatic couplings. In this region, the main process is dissociation through Rydberg states. For these states, the interplay between the chosen value of $\delta$ in the ROM definition, the energy spacing resulting from discretization in the finite box, and the pulse duration determine the accuracy of the results. For instance, as can be seen in Fig. 9(a), there is no difference between total and differential ROM probabilities when a shorter pulse is used, because in this case the relevant Rydberg states do not need to be resolved individually due to the relatively large laser energy bandwidth. Since the present work focuses on ionization and the proper description of Rydberg states needs a specific treatment (for this work and any other method), it is not further discussed in the present work. In any case, it must be pointed out that, in the energy region where ionization is the dominant channel, the results obtained from the differential and the total ROM are indistinguishable. The same occurs in the region where the lower bound excited states, like the $2 p \sigma_{u}$ one, are populated. These results prove that ROM spectra, in which the total energy is split into electronic and energy parts, are accurate and that the normalization of both differential and total ROM is correct.

\section{CONCLUSIONS}

We have presented a method to extract correlated photoelectron- and nuclear-kinetic energy spectra for molecules interacting with strong, ultrashort laser fields in the SAE approximation. We have focused on $\mathrm{H}_{2}{ }^{+}$dissociation and photoionization by femtosecond laser pulses in the XUV-IR frequency range.

We have extended the ROM from atoms [37,38] to molecules [16]. For this, we solved the TDSE, by using a grid method, in a $(1+1) \mathrm{D}$ model for the $\mathrm{H}_{2}{ }^{+}$molecule with one degree of freedom for the electron and another one for the nuclei. After interaction with the laser field, the final wave function is analyzed by means of a resolvent operator which is a direct product of two operators, one that selects the electronic state and electronic energy and another one that selects the nuclear energy. The modulus square of the projected wave function is the doubly differential probability density, after multiplication by a constant. In the selection of the electronic state, we use the potential energy curves calculated in the BO approximation, but not in the time propagation. If the resolvent operator was applied to the final wave function at infinite time, the analysis would be exact.

This differential ROM provides correlated photoelectronand nuclear-kinetic energy spectra (CKE) in which the energy sharing between the fragments can be analyzed. In particular, we have shown results for few-photon absorption, for boundbound electronic transitions, and for strong IR fields, in which multiphoton absorption and tunneling are present. In the latter case, we have shown how the differential ROM makes it possible to track the transition between both regimes. We have also shown isotopic effects, comparing $\mathrm{H}_{2}{ }^{+}$and $\mathrm{D}_{2}{ }^{+}$ ionization for different pulses.

Finally, we have compared the results obtained by integrating the differential probabilities with those obtained by using the total ROM in which only one operator containing the total molecular Hamiltonian is used. The agreement between results obtained with both methods is excellent, showing that (i) the approximation made by projecting the wave function at finite time, in the differential ROM, is justified and (ii) the normalization used to obtain the probabilities is correct. Nevertheless, since $\mathrm{H}_{2}{ }^{+}$is a rather unique molecule because of its very simple electronic structure, it would be desirable to check the performance of the ROM method in more complex molecular systems. In particular, the accuracy of the method must be checked in the ionization threshold region, where nonadiabatic couplings may be involved.

\section{ACKNOWLEDGMENTS}

This work was accomplished with an allocation of computer time from Mare Nostrum BSC and CCC-UAM and was partially supported by the MICINN Projects No. FIS201015127 and No. CSD 2007-00010, ERA-Chemistry Project No. PIM2010EEC-00751, the European grants No. MC-ITN CORINF and No. MC-RG ATTOTREND, the European COST Action No. CM0702, and European Research Council Advanced Grant No. XCHEM 290853. R.E.F.S. acknowledges a Ph.D. contract from ITN CORINF and Grant No. SFRH/BD/84053/2012 from the Portuguese government. P.R. acknowledges a Juan de la Cierva contract grant from the Spanish MICINN. The authors also thank Professor L. B. Madsen for kindly sending us the numerical data used in Appendix C. 


\section{APPENDIX A: TECHNICAL DETAILS}

In a grid approach, the molecular wave function $\Psi(z, R)$ is spatially discretized. In the present work, a homogeneous grid for both coordinates $(z$ and $R$ ) has been used, so that the wave function becomes a $2 \mathrm{D}$ array $\Psi\left(z_{i}, R_{j}\right)=\Psi_{i j}$. The number of $i$ and $j$ points define the spatial resolution for a given box size. The kinetic energy operators are approximated by using a finite difference method of the second order and the wave function is forced to be zero at the edge of the box, so that the kinetic energy operators are described by tridiagonal matrices. For example, the electronic energy operator will couple the $(z, R)$ point to the $(z+\Delta z, R)$ and $(z-\Delta z, R)$ points, and the corresponding matrix is symmetric, implying that the global Hamiltonian is Hermitian and the usual split-operator and Crank-Nicolson techniques can be used for the time propagation.

We have seen in Sec. III A that the resolvent operator is written as a product of Green's operators. For the electronic resolvent operator we have

$$
\begin{gathered}
\hat{R}_{e l}=\prod_{j=1}^{n} \delta_{e l} \hat{G}_{e l}\left(\varepsilon_{e l}-q_{j} \delta_{e l}\right), \\
\hat{G}_{e l}\left(\varepsilon_{e l}-q_{j} \delta_{e l}\right)=\frac{1}{\varepsilon_{e l}-\hat{H}_{e l}+E_{k}(\boldsymbol{R})-q_{j} \delta_{e l}} .
\end{gathered}
$$

The inverse matrix of the electronic Green's operator is tridiagonal for a fixed value of the internuclear distance. So in order to calculate the action of the electronic Green's operator on the wave function, we only need to solve a system of linear equations associated with a tridiagonal matrix. We solve this system by using the Thomas algorithm [51] at each value of the internuclear distance. The same method is used for the nuclear resolvent by fixing the electronic coordinate.

\section{APPENDIX B: CONVERGENCE OF THE CALCULATIONS}

As described in the main text, we have checked the convergence of the results with respect to several parameters. In all cases, a change of about $30 \%$ in these parameters leads to results practically indistinguishable from those shown in the paper. Here we only illustrate how the use of the $\mathrm{BO}$ approximation in the definition of the resolvent operator affects the results. As explained in the text, this approximation becomes exact for the dissociative ionization channel when $T_{\text {prop }} \rightarrow \infty$. Thus, to check the accuracy of the results, we have performed the ROM analysis at increasingly longer times after the pulse, which implies integrating the TDSE further and further in time.

In Fig. 10 the photoelectron spectra calculated at the end of the pulse (black line), at $2.57 \mathrm{fs}$ (red line), and at $5.14 \mathrm{fs}$ (blue line) after the end of the pulse are presented. As can be seen, the photoelectron and KER spectra are practically converged at the end of the pulse. Very tiny differences are only observed at the highest photoelectron energies. This is easy to understand since dissociation of $\mathrm{H}_{2}{ }^{+}$occurs within a few fs, i.e., within the pulse duration.

In Fig. 11, the total energy spectra calculated with the total ROM (black line) and the differential ROM applied at the end
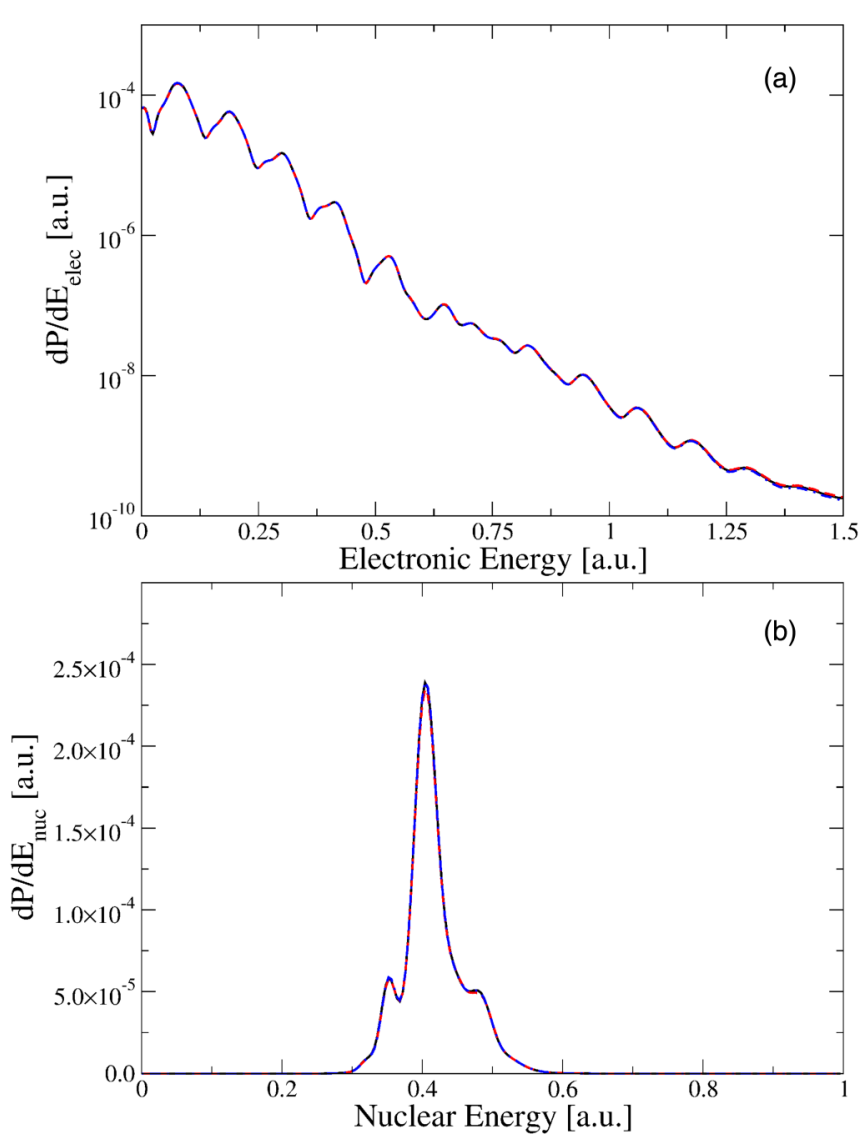

FIG. 10. (Color online) Photoelectron spectra (top) and nuclearkinetic energy spectra (bottom) for a 400-nm pulse with $I=$ $10^{14} \mathrm{~W} / \mathrm{cm}^{2}$ and $T=16 \mathrm{fs}$. ROM analysis performed at the end of the pulse (black solid line), $2.57 \mathrm{fs}$ after the end of the pulse (red dashed line), and $5.14 \mathrm{fs}$ after the end of the pulse (blue dash-dotted line).

of the pulse (red line) and 5.14 fs after the pulse (blue line) are presented. We see that the results obtained from the differential ROM are already converged when probabilities are extracted

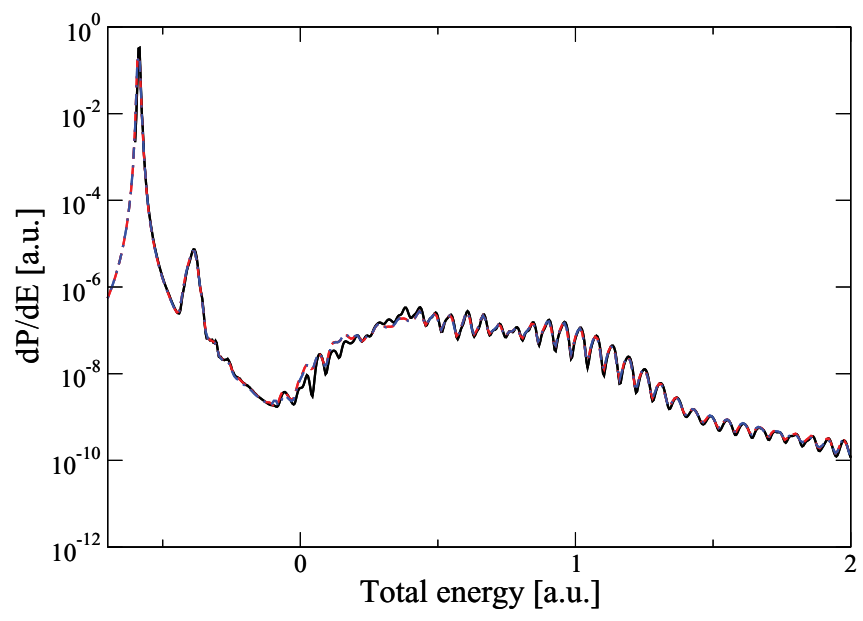

FIG. 11. (Color online) Total energy spectra for a $800 \mathrm{~nm}$ pulse with $I=10^{14} \mathrm{~W} / \mathrm{cm}^{2}$ and $T=32$ fs. Total ROM analysis is performed at the end of the pulse (black solid line) and differential ROM analysis is performed at the end of the pulse (red dashed line) and $5.14 \mathrm{fs}$ after the end of the pulse (blue dash-dotted line). 


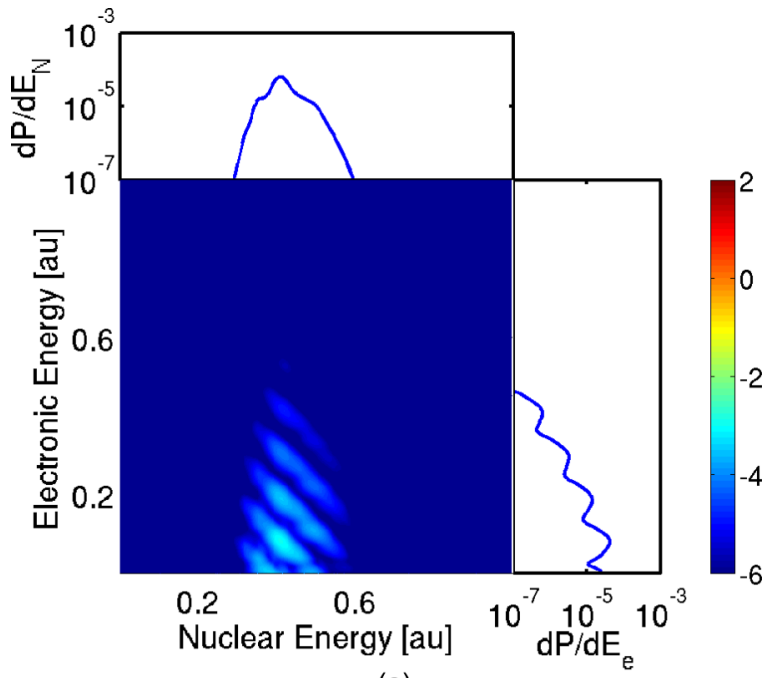

(a)

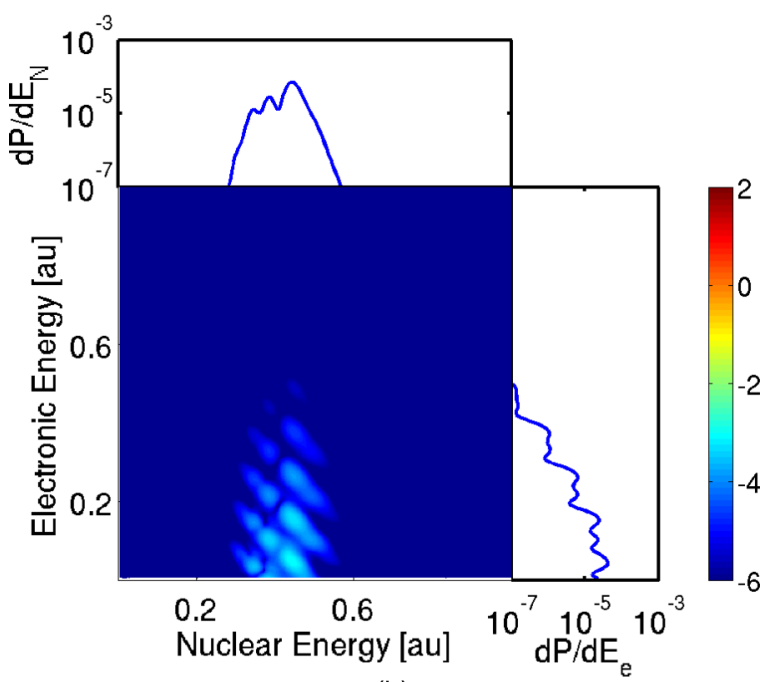

(b)

FIG. 12. (Color online) Comparison of our calculations using the ROM (a) and data extracted from Madsen et al. [19] (b). The data of Madsen et al. have been multiplied by a global coefficient equal to 0.10098 . The laser peak intensity is set to $8.8 \times 10^{13} \mathrm{~W} / \mathrm{cm}^{2}$ and the central wavelength to $400 \mathrm{~nm}$. The pulse duration is of 10 optical cycles and the $\mathrm{H}_{2}{ }^{+}$initial state is $v=0$.

at the end of the pulse. These results are indistinguishable from those obtained from the total ROM, except for energies in the interval 0-0.5 a.u., where small differences are observed (see main text).

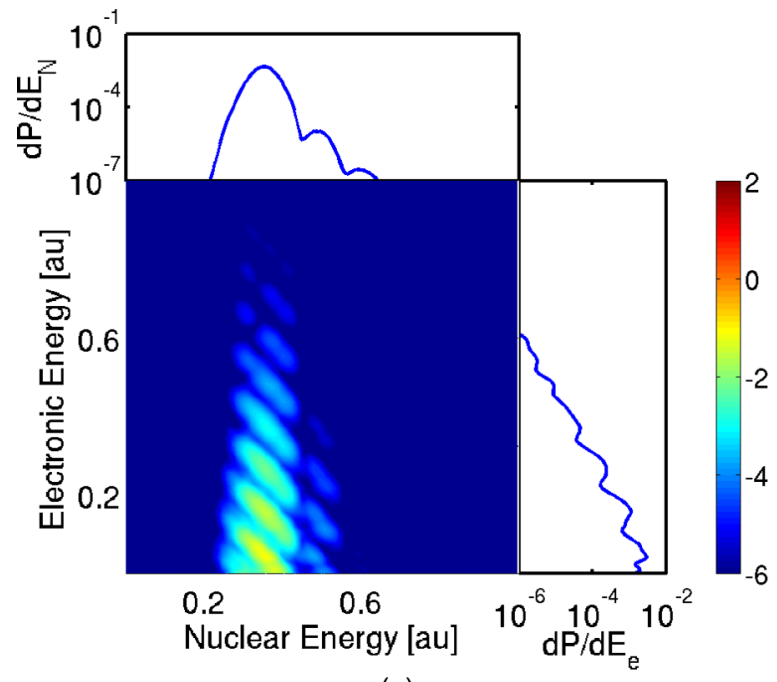

(a)

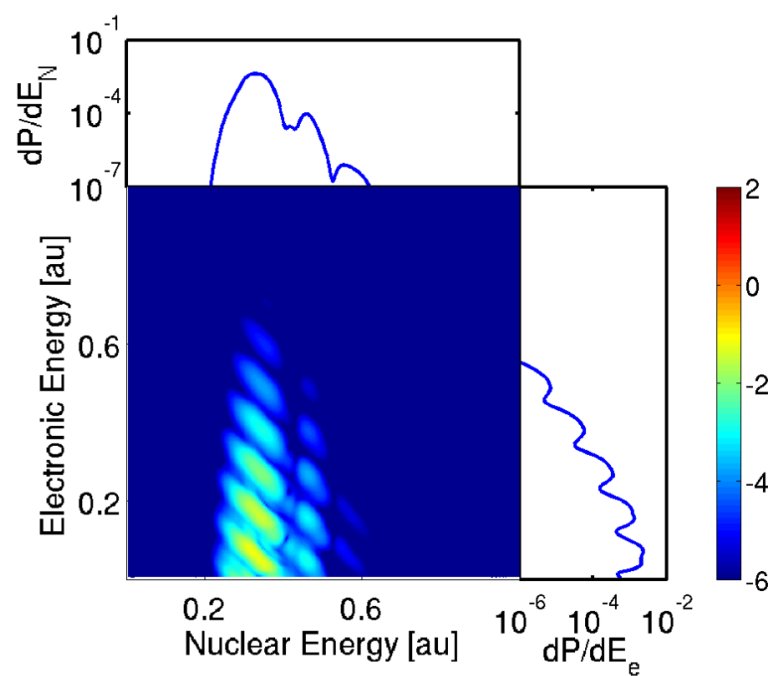

(b)

FIG. 13. (Color online) Same as in Fig. 12, but for an $\mathrm{H}_{2}{ }^{+}$initial state equal to $v=2$ and the data from [19] (b) are multiplied by 0.33502 .

\section{APPENDIX C: COMPARISON WITH PREVIOUS DATA}

In this Appendix we compare the results obtained from the ROM method to the data taken from [19] (see Figs. 12 and 13). The laser parameters are the same as in Ref. [19].
[1] P. H. Bucksbaum, A. Zavriyev, H. G. Muller, and D. W. Schumacher, Phys. Rev. Lett. 64, 1883 (1990).

[2] L. J. Frasinski, J. H. Posthumus, J. Plumridge, K. Codling, P. F. Taday, and A. J. Langley, Phys. Rev. Lett. 83, 3625 (1999).

[3] T. Zuo and A. D. Bandrauk, Phys. Rev. A 52, R2511 (1995).

[4] A. D. Bandrauk, Atoms in Strong Fields (Marcel Dekker, New York, 1994).

[5] A. Giusti-Suzor, F. H. Mies, L. F. DiMauro, E. Charron, and B. Yang, J. Phys. B 28, 309 (1995).
[6] J. H. Posthumus, Rep. Prog. Phys. 67, 623 (2004).

[7] O. Smirnova, Y. Mairesse, S. Patchkovskii, N. Dudovich, D. Villeneuve, P. B. Corkum, and M. Y. Ivanov, Nature (London) 460, 972 (2009).

[8] Y. Mairesse, A. de Bohan, L. J. Frasinski, H. Merdji, L. C. Dinu, P. Monchicourt, P. Breger, M. Kovačev, R. Taïeb, B. Carré, H. G. Muller, P. Agostini, and P. Salières, Science 302, 1540 (2003).

[9] I. Ben-Itzhak, P. Q. Wang, J. F. Xia, A. M. Sayler, M. A. Smith, K. D. Carnes, and B. D. Esry, Phys. Rev. Lett. 95, 073002 (2005). 
[10] D. Toffoli, R. R. Lucchese, M. Lebech, J. C. Houver, and D. Dowek, J. Chem. Phys. 126, 054307 (2007).

[11] M. Lewenstein, P. Balcou, M. Y. Ivanov, A. L'Huillier, and P. B. Corkum, Phys. Rev. A 49, 2117 (1994).

[12] J. Itatani, J. Levesque, D. Zeidler, H. Niikura, H. Pépin, J. C. Kieffer, P. B. Corkum, and D. M. Villeneuve, Nature (London) 432, 867 (2004).

[13] C. Vozzi, M. Negro, F. Calegari, G. Sansone, M. Nisoli, S. De Silvestri, and S. Stagira, Nat. Phys. 7, 822 (2011).

[14] R. E. F. Silva, P. Rivière, and F. Martín, Phys. Rev. A 85, 063414 (2012).

[15] A. González-Castrillo, A. Palacios, F. Catoire, H. Bachau, and F. Martín, J. Phys. Chem. A 116, 2704 (2012).

[16] R. E. F. Silva, F. Catoire, P. Rivière, H. Bachau, and F. Martín, Phys. Rev. Lett. 110, 113001 (2013).

[17] H. Stapelfeldt, E. Constant, and P. B. Corkum, Phys. Rev. Lett. 74, 3780 (1995).

[18] M. Magrakvelidze, C. M. Aikens, and U. Thumm, Phys. Rev. A 86, 023402 (2012).

[19] C. B. Madsen, F. Anis, L. B. Madsen, and B. D. Esry, Phys. Rev. Lett. 109, 163003 (2012).

[20] A. Staudte et al., Phys. Rev. Lett. 98, 073003 (2007).

[21] S. Chelkowski, A. D. Bandrauk, A. Staudte, and P. B. Corkum, Phys. Rev. A 76, 013405 (2007).

[22] B. D. Esry, A. M. Sayler, P. Q. Wang, K. D. Carnes, and I. Ben-Itzhak, Phys. Rev. Lett. 97, 013003 (2006).

[23] B. D. Esry and I. Ben-Itzhak, Phys. Rev. A 82, 043409 (2010).

[24] H. Niikura, H. Légaré, R. Hasbani, A. D. Bandrauk, M. Y. Ivanov, D. M. Villeneuve, and P. B. Corkum, Nature (London) 417, 917 (2002).

[25] A. D. Bandrauk and S. Chelkowski, J. Mol. Struct.: THEOCHEM 591, 199 (2002).

[26] P. Moreno, L. Plaja, and L. Roso, Laser Phys. 7, 602 (1997).

[27] C. B. Madsen, M. Abu-samha, and L. B. Madsen, Phys. Rev. A 81, 043413 (2010).

[28] M. Winter, R. Schmidt, and U. Thumm, New J. Phys. A 12, 023020 (2010).

[29] M. Fischer, U. Lorenz, B. Schmidt, and R. Schmidt, Phys. Rev. A 84, 033422 (2011).

[30] I. Sánchez and F. Martín, Phys. Rev. Lett. 79, 1654 (1997).
[31] F. Martín, J. Fernández, T. Havermeier, L. Foucar, Th. Weber, K. Kreidi, M. Schöffler, T. Jahnke L. Schmidt, O. Jagutzki, A. Czasch, E. P. Benis, T. Osipov, A. L. Landers, A. Belkacem, M. H. Prior, H. Schmidt-Böcking, C. L. Cocke, and R. Dörner, Science 315, 629 (2007).

[32] J. Ullrich, R. Moshammer, R. Dörner, L. Ph.H. Schmidt, and H. Schmidt-Böcking, Rep. Prog. Phys. 66, 1463 (2003).

[33] P. Rivière, R. E. F. Silva, and F. Martín, J. Phys. Chem. A 116, 11304 (2012).

[34] J. Wu, M. Kunitski, M. Pitzer, F. Trinter, L. Ph. H. Schmidt, T. Jahnke, M. Magrakvelidze, C. B. Madsen, L. B. Madsen, U. Thumm, and R. Dörner, Phys. Rev. Lett. 111, 023002 (2013).

[35] H. Bachau, E. Cormier, P. Decleva, J. Hansen, and F. Martín, Rep. Prog. Phys. 64, 1815 (2001).

[36] J. Javanainen, J. H. Eberly, and Q. Shu, Phys. Rev. A 38, 3430 (1988)

[37] K. J. Schafer and K. C. Kulander, Phys. Rev. A 42, 5794 (1990).

[38] F. Catoire and H. Bachau, Phys. Rev. A 85, 023422 (2012).

[39] P. Colosimo, G. Doumy, C. I. Blaga, J. Wheeler, C. Hauri, F. Catoire, J. Tate, R. Chirla, A. M. March, G. G. Paulus, H. G. Muller, P. Agostini, and L. F. DiMauro, Nat. Phys. 4, 386 (2008).

[40] M. Awasthi, Y. V. Vanne, A. Saenz, A. Castro, and P. Decleva, Phys. Rev. A 77, 063403 (2008).

[41] D. J. Tannor, Introduction to Quantum Mechanics a TimeDependent Perspective (University Science Book, South Orange, NJ, 2007).

[42] B. Feuerstein and U. Thumm, Phys. Rev. A 67, 043405 (2003).

[43] L. D. Landau and E. M. Lifshitz, Quantum Mechanics, Nonrelativistic Theory (Pergamon, Oxford, UK, 1965).

[44] M. Forre and H. Bachau, Phys. Rev. A 77, 053415 (2008).

[45] H. D. Cohen and U. Fano, Phys. Rev. 150, 30 (1966).

[46] D. M. Chase, Phys. Rev. 104, 838 (1956).

[47] A. Palacios, H. Bachau, and F. Martín, Phys. Rev. A 74, 031402 (2006).

[48] H. M. Tetchou Nganso, Yu. V. Popov, B. Piraux, J. Madroñero, and M. G. Kwato Njock, Phys. Rev. A 83, 013401 (2011).

[49] B. Feuerstein and U. Thumm, J. Phys. B 36, 707 (2003).

[50] R. S. Berry and S. E. Nielsen, J. Chem. Phys. 49, 116 (1968).

[51] S. D. Conte, Elementary Numerical Analysis: An Algorithmic Approach (McGraw-Hill College, New York, 1980). 